San Jose State University

SJSU ScholarWorks

Master's Theses

Master's Theses and Graduate Research

1990

\title{
Light-induced electron spin resonance in hydrogenated amorphous silicon nitride
}

Eric D. Tober

San Jose State University

Follow this and additional works at: https://scholarworks.sjsu.edu/etd_theses

\section{Recommended Citation}

Tober, Eric D., "Light-induced electron spin resonance in hydrogenated amorphous silicon nitride" (1990). Master's Theses. 3298.

DOI: https://doi.org/10.31979/etd.9med-unr5

https://scholarworks.sjsu.edu/etd_theses/3298

This Thesis is brought to you for free and open access by the Master's Theses and Graduate Research at SJSU ScholarWorks. It has been accepted for inclusion in Master's Theses by an authorized administrator of SJSU ScholarWorks. For more information, please contact scholarworks@sjsu.edu. 


\section{INFORMATION TO USERS}

The most advanced technology has been used to photograph and reproduce this manuscript from the microfilm master. UMI films the text directly from the original or copy submitted. Thus, some thesis and dissertation copies are in typewriter face, while others may be from any type of computer printer.

The quality of this reproduction is dependent upon the quality of the copy submitted. Broken or indistinct print, colored or poor quality illustrations and photographs, print bleedthrough, substandard margins, and improper alignment can adversely affect reproduction.

In the unlikely event that the author did not send UMI a complete manuscript and there are missing pages, these will be noted. Also, if unauthorized copyright material had to be removed, a note will indicate the deletion.

Oversize materials (e.g., maps, drawings, charts) are reproduced by sectioning the original, beginning at the upper left-hand corner and continuing from left to right in equal sections with small overlaps. Each original is also photographed in one exposure and is included in reduced form at the back of the book.

Photographs included in the original manuscript have been reproduced xerographically in this copy. Higher quality $6^{\prime \prime} \times 9^{\prime \prime}$ black and white photographic prints are available for any photographs or illustrations appearing in this copy for an additional charge. Contact UMI directly to order.

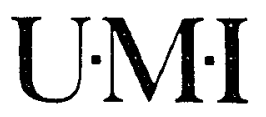

Universily Microfims international

A Bell \& Howel! Intormation Company

300 Norin Zeeb Road. Ann Arbor. MI 48106-1346 USA

$313761.4700 \quad 800521.0600$ 
Light-induced electron spin resonance in hydrogenated amorphous silicon nitride

\author{
Tober, Eric D., M.S.
}

San Jose State University, 1990

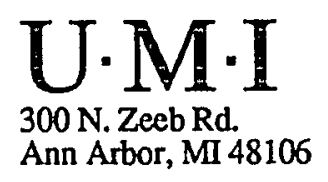




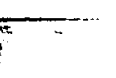




\title{
Light-Induced Electron Spin Resonance in Hydrogenated Amorphous Silicon Nitride
}

\author{
A Thesis \\ presented to \\ The Faculty of the Department of Physics \\ San Jose State University \\ In Partial Fulfillment \\ of the Requirements for the Degree \\ Master of Science
}

by

Eric D. Tober

May, 1990 


\section{APPROVED FOR THE DEPARTMENT OF PHYSICS}
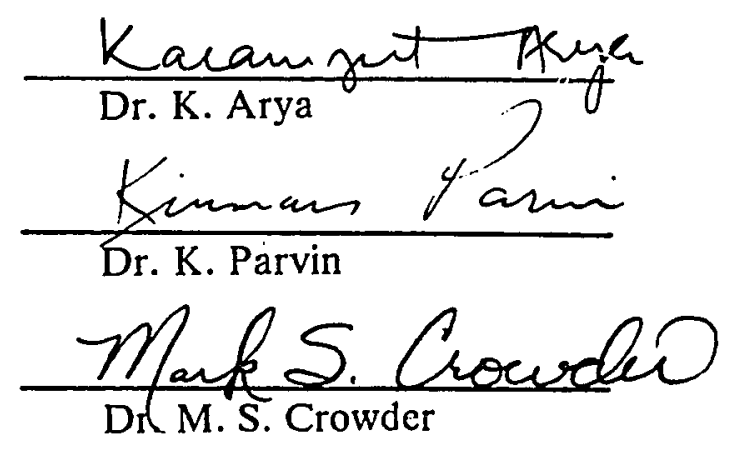

\section{APPROVED FOR THE UNIVERSITY}

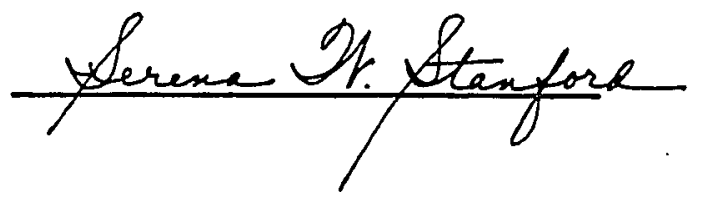




\section{ABSTRACT \\ LIGHT-INDUCED ELECTRON SPIN RESONANCE IN HYDROGENATED AMORPHOUS SILICON NITRIDE \\ by Eric D. Tober}

Hydrogenated, amorphous silicon nitride (a-SiN:H) is used extensively in semiconductor device technology. It is commonly used as a gate dielectric in a-Si:H thin film transistors and as a passivation layer for integrated circuits. When exposed to light, defects are induced in the material which have been associated with under-coordinated Si dangling bonds. The light-induced defects are capable of trapping mobile charges and causing a degradation in the electrical properties of the material. This, of course, would adversely affect device stability due to charge accumulating in the nitride. Therefore, it is of importance to understand the propertics of these light-induced defects and their behavior under illumination.

Electron spin resonance (ESR) spectroscopy is an excellent method for monitoring paramagnetic centers in insulating materials. The light-induced defects are paramagnetic in the electrically neutral state and are detectable using ESR. ESR is used to study the production and photo-bleaching of neutral defects as a function of illumination time, photon energy and temperature. Included in this thesis is a physical model to explain the light-induced phenomena. 


\section{Table of Contents}

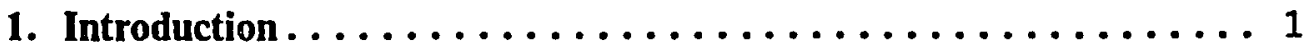

2. Amorphous Semiconductors..................... 4

2.1 The Energy Gap......................... 4

2.2 Dangling Bonds.......................... 5

2.3 Floating Bonds $\ldots \ldots \ldots \ldots \ldots \ldots \ldots \ldots \ldots \ldots, 7$

2.4 Correlation Energy $\ldots \ldots \ldots \ldots \ldots \ldots \ldots \ldots \ldots, 7$

2.5 Light-Induced Defects..................... 8

2.6 Multiple Trapping and Dispersive Transport ......... 10

3. Electron Spin Resonance.$\ldots \ldots \ldots \ldots \ldots \ldots \ldots \ldots \ldots \ldots$. 12

3.1 ESR Theory $\ldots \ldots \ldots \ldots \ldots \ldots \ldots \ldots \ldots \ldots \ldots \ldots, 12$

3.2 Hyperfine Interaction...................... 16

3.3 The ESR Spectrometer.................... 17

4. Light-Induced Electron Spin Resonance

in Hydrogenated Amorphous Silicon Nitride............. 19

4.1 Experimental Procedure..................... 20

4.2 Production of the LESR..................... 22

4.3 Photo-Bleaching of the LESR.................. 27

4.4 Charged Defect Production.................... 34

5. Summary $\ldots \ldots \ldots \ldots \ldots \ldots \ldots \ldots \ldots \ldots \ldots \ldots, \ldots \ldots \ldots$

Acknowledgements........................... 39

References................................. 40

Figure Captions $\ldots \ldots \ldots \ldots \ldots \ldots \ldots \ldots \ldots \ldots \ldots \ldots \ldots, 45$

Tables................................... 48

Figures $\ldots \ldots \ldots \ldots \ldots \ldots \ldots \ldots \ldots \ldots \ldots, 50$ 


\section{Introduction}

Amorphous silicon based matcrials play an important role in semiconductor device technology. Such materials are commonly used in thin film transistors, diodes, and photovoltaic cells. In particular, hydrogenated amorphous silicon nitride $(\mathrm{a}-\mathrm{SiN}: \mathrm{H})$ is used as a passivation layer for the protection of semiconductor devices. Furthermore, this material is commonly used as a gate dielectric in thin film a-SiN:H based transistors. Unfortunately, there exists a number of defects in a-SiN:H after deposition. These defects have been attributed to undercoordinated $\mathrm{Si}$ atoms (dangling bonds) or over-coordinated $\mathrm{Si}$ atoms (floating bonds) existing in the bulk of the material. These defects are capable of existing in one of three possible charged states: neutral $\left(D^{\circ}\right)$, positive $\left(D^{+}\right)$, and negative (D-). Upon exposure to light, the density of neutral defects increases dramatically. These neutral defects trap mobile carriers as they diffuse through the material. The trapping of charge in the nitride has an important effect on any device applications. As the trapped charge increases in the nitride gate dielectric, shifts can occur in the current-voltage thresholds of a thin film transistor (TFT). This, of course, is undesirable since the device performance is no longer stable. Currently, a-SiN:H is incorporated into a number of devices which must operate while under exposure to light (photovoltaic cells ${ }^{40}$ and TFT's that act as switching slements in LCD displays ${ }^{41-44}$ ). Therefore, it is important to characterize the behavior of this material when exposed to light and to understand the physical processes involved in the defect production. The kinetics of the production of the neutral defects $\left(D^{\circ}\right)$ under illumination has been examined in an attempt to attribute a microscopic process to the defect creation. Currently, it is uncertain 
as to whether the light-induced defects (neutral and charged) are inherent to the material as deposited or created by the illumination. A small number $\left(\sim 10^{16} \mathrm{~cm}^{-3}\right)$ of neutral defect arc present before illumination indicating that a fraction of the defects (if not all) observed after illumination are inherent to the material.

The neutral defects $\left(D^{\circ}\right)$ contain one unpaired elcctron, making them paramagnetic. The charged states, however, contain no unpaired electrons and are therefore diamagnetic. Electron spin resonance (ESR) spectroscopy is an excellent method for monitoring exclusivcly the generation of the neutral, trapping defects. ESR spectroscopy offers a non-intrusive, accurate measure of the density of paramagnetic centers. ESR is due to the interaction of the microwave electro-magnetic energy with the paramagnetic centers in which a resonance conciitisn is set up between Zeeman split electron spin levels. Microwaves are used to induce quantum mechanical transitions between the spin quantum levels, resulting in the resonant absorption of the microwaves and a corresponding ESR signal. The ESR signal is indicative of the specific paramagnetic center and can be used to determine the density of the neutral defects $\left(D^{\circ}\right)$.

The main purpose of this project is to explore the effects of light on the defect states in a-SiN:H using ESR spectroscopy. Specifically, the photon energy dependence for neutral defect production and photo-bleaching, the temperature dependence of the bleaching, and the thermal decay of the produced LESR at room temperature has been determined. Also, a mathematical model is proposed to describe the kinetics of the neutral and charged defect production as well as the observed bleaching phenomenon. This work was performed at I.B.M. San Jose, 
5600 Cottle Rd. under the supervision of Dr. Mark S. Crowder. Preliminary reports on this work were made at the March (1990) APS conference and the April (1990) MRS conference. 


\section{Amorphous Semiconductors}

Amorphous semiconductors lack the periodic arrangement of atoms that characterizes their crystalline analogs. This leads to some fundamental differences in the electrical properties of these materials. The lack of periodicity also makes it difficult to model mathematically the energy gap, density of states, etc. for these materials. This is due to fact that one cannot use many of the simplifications that are used for periodic, crystalline structures $\$ .2$ It is important to note, however, that the amorphous semiconductors still retain many of the properties found in the crystalline state. One of the most important of these is that they have a distinct energy gap with a conduction band, valence band, and Fermi en-

ergy. Also, they may be either $\mathrm{p}$ or $\mathrm{n}$ doped which allows them to be used in current device technology. These materials can be produced in thin films with high purity by such methods as glow discharge ${ }^{2}$, chemical vapor deposition $\left(C V D^{1,2}\right)$, and plasma enhanced chemical vapor deposition (PECVD). Other methods of preparation include slow cooling from the melt and ion bombardment.

\subsection{The Energy Gap}

Even though amorphous semiconductors lack the long range periodic ordering of crystals, they retain distinct encrgy gaps. This arises from the short range ordering still present in the molecular arrangement of the atoms. In other words 
the average bond angles, bond lengths, and coordination between atoms still follows closely the values found in crystals. However, there exists a distribution of possible bond angles and bond lengths. This causes the valence and conduction band edges to tail into the energy gap in an exponential fashion ${ }^{1,2}$ (Fig. 2.1). These tail states are localized duc to the disorder of the atomic arrangements (Ancierson localization ${ }^{2}$ ). The localized states have an important effect upon the electrical properties of the amorphous semiconductors. Mobile carriers cail be trapped at these sites and re-excited, leading to dispersive transpoi: ${ }^{2,3}$.

Other states exist in the energy gap besides the localized band tails. These states arise from structural defects in the arrangement of atoms due to the nonperiodicity of the amorphous system. The defect states lie near miugap in a-Si (amorphous silicon). The rest of this chapter will be concerned with only a-Si based materials. Two possible defect configurations have been proposed as the source of these states: the silicon dangling bond and the silicon floating bond.

\subsection{Dangling Bonds}

The Si dangling bond consists of a Si atom covalently bonded to three other atoms instead of four. This undcrcoordination of the $\mathrm{Si}$ atom $\mathrm{z}$-ises due to the local configurations about the atom. For a normally bonded $\mathrm{Si}$ atom, there are four $\mathrm{sp}^{3}$ hybrid valence electron orbitals involved in the tetrahcdral covalent bonding. A silicon dangling bond occurs when only three neighbor atoms are available for bonding. In this case, three orbitals from the neighbors form bonding and antibonding states with three out of the four possible hybrid states 
of the Si atom. The fourth state remains unbonded and is the Si dangling bond (Fig. 2.2a) This dangling bond is capable of containing 0,1 or 2 electrons. The configuration of the undercoordinated $\mathrm{Si}$ atoms leads energetically to localized states existing in the energy gap ${ }^{5-9}$. The Si dangling bonds are capable of existing in one of three possible charge states: neutral $\left(D^{\circ}\right)$, negative $\left(D^{-}\right)$, and positive (D'). The neutral dangling bond contains the one excess valence electron not involved in the bonding to nearest neighbors. This unpaired electron gives the state a net electronic spin and gives rise to paramagnetism. The positive dangling bond has given up its excess valence electron and is therefore diamagnetic. In a similar manner, the negative dangling bond has captured a second electron and therefore contains an electron pair with no net spin (diamagnetic).

In support of this model for the defects, it has been shown that the incorporation of hydrogen into the a-Si based materials severely reduces the number of defect states in the gap. It is belicved that atomic hydrogen bonds to the undercoordinated Si atoms and thereby passivates the dangling bonds and removes them from the energy gap. Also, hydrogen bonds allow the amorphous structure to be less rigid. This is due to the fact that hydrogen coordinates with only one other atom, allowing a greater frecdom in configuration for the atoms it is bonded to. Hydrogenation is extremely important for a-Si since it lowers the defect level enough to enable doping and, therefore, use in semiconductor devices. 


\subsection{Floating Bonds}

Another explanation for the existence of defect states in a-Si based materials has been set forth by Pantelides ${ }^{4}$. In this model, it is proposed that overcoordination and not undercoordination is the source of the observed defect states. In other words, the $\mathrm{Si}$ atom that is the site of the defect is bonded to five neighbor atoms (Fig. 2.2b) and not three as in the dangling bond case. These fivecoordinate Si atoms give rise to states labeled as "floating bonds." Overcoordinated $\mathrm{Si}$ atoms have a similar formation energy to the dangling bonds ${ }^{11}$ and therefore may exist.

\subsection{Correlation Energy}

Correlation energy is the measure of a given state's affinity for capturing an electron. In crystalline band theory, the one-electron model for the hamiltonian does not take into account interactions with other electrons in the lattice. Therefore, interaction of electrons in the same localized state is not accounted for. The difference between the expected one-electron ground state energy and the actual energy with electron interactions is the correlation energy (U). If a given state has a negative $U$, then it has an affinity for capturing an electron. The correlation energy could possibly be used to distinguish between the two types of defects previously mentioned. Pantclides sup 4 states that the the correlation energy for the floating bond in the neutral charge state should be positive $(\sim 0.3$ $\mathrm{eV}$ ) since the five coordinate atom is bonded to its five neighbors by less than 2 electrons per bond (as is the case for deep substitutional impurities). If this is the 
case, then the ground state of the defect in a-Si based materials would be the neutral $\left(D^{\circ}\right)$ state or the positive $\left(D^{+}\right)$state. Calculations by Bar-Yam et allindicate that the correlation energy for the neutral dangling bond should be negative and, therefore, have a negatively charged ground state $\left(D^{-}\right.$state -- doubly occupied by electrons). However, distortions of the local atomic arrangements abollt the dangling bond were able to produce a small positive $U(0.05 \mathrm{eV})$. Since there exists a distribution of bond angles and lengths in the amorphous network, there may exist a small population of dangling bonds with a positive $U$.

\subsection{Light-Induced Defects}

It has been previously observed by Staebler and Wronski ${ }^{13}$ that light induces changes in the electrical properties of a-Si:H. This effect has been shown to arise from the generation of metastable defects which act as trapping centers for mobile carriers and has been observed in a number of a-Si based alloys ${ }^{7,8,14,23,45}$. In conjunction with this effect, it is observed that the number of paramagnetic defects in a-Si:H increase significantly (about 100 times as measured by EPR) upon illumination. This is also the case with hydrogenated, amorphous silicon nitride (a-SiN:H). In addition to the increase in the neutral defects in the nitride, it has been observed that the number of positively charged $\left(\mathrm{D}^{+}\right)$defects increase under illumination ${ }^{17}$. Krick et al ${ }^{16}$ showed that the neutral defects were responsible for the observed charge trapping in a-SiN:H. These defects are metastable and can be thermally annealed at room temperature and above ${ }^{18,26}$. 
Controversy exists as to the origin of the light-induced defects. Stutzman et all ${ }^{14}$ proposed that Si-Si weak bonds are broken when the material is exposed to light. Photo-excited carriers recombine non-radiatively at the site of the weak bond (a Si-Si bond that is stretched, lowering the bond energy) and fracture the bond, producing two dangling bonds in its place. Hence, the observed increase in the $D^{\circ}$ defects is due to the overall increase in the total number of defects $\left(D^{o}+D^{-}+D^{+}\right)$. This increase in trapping centers (defects) is then responsible for the Staebler-Wronski effect. It is important to note that in this model the state prior to illumination (ground state) of the light induced defect is a weak Si-Si bond and that the metastable, light-induced defect is a Si dangling bond. Another model for the Staebler-Wronski effect was put forth by Adler ${ }^{19}$. In this model the ground state of the light induced $D^{\circ}$ defect is one of the charged defects $\left(D^{+}, D^{-}\right)$. The charged states have large trapping cross sections for electrons and holes, respectively. Under illumination, photo-excited electrons (holes) are trapped by the positively (negatively) charged defects converting them into metastable, neutral defects. Under this process, no new defects are formed by bond breaking. Each of these processes can be represented by the same two level energy-configuration diagram (Fig. 2.3). In this diagram, $\mathrm{V}_{0}$ is the energy barrier between the two configurational states $q_{0}$ (the ground state) and $q_{1}$ (the metastable state). Under illumination, if

$$
h v \geq E_{n}
$$


where $h v$ is the photon energy then transitions from the $q_{0}$ state to the $q_{1}$ state and vice versa are possible if $n=1$. However, only transitions from the $q_{1}$ state to the $q_{0}$ state are possible if $n=2$. All states in $q_{1}$ with potential energy

$$
V_{o}\left(q_{1}\right)>>k_{b} T
$$

remain in the metastable state after the illumination is ended (Stutzman et al ${ }^{14}$ ). The metastable states can be removed by thermal excitation with energies on the order of $V_{0}$ and optical excitations of energy greater than $E_{2}$. This is extremely important for understanding the phenomenon reported in the Section 4.3 (Photo-bleaching of the LESR).

\subsection{Multiple Trapping and Dispersive Transport}

Carrier transport in amorphous semiconductors is strongly dispersive in nature. The physical origin of the dispersive transport arises from the presence of localized states (band tails and defects) in the energy gap. Two possible mechanisms have been proposed to explain the observed dispersion. In the first model, mobile carriers are trapped by localized states in the gap and subsequently reexcited into extended states for a number of discrete steps (Fig. 1.4). In this case, the presence of the localized states impedes the carriers as they diffuse through the material (multiple trapping ${ }^{3,15}$ ). The second process that may give rise to the dispersion of the carriers involves the tunneling of electrons (or holes) between the localized states (Fig. 2.4). Tunneling would occur when an electron in a localized 
state below the Fermi energy tunnels to a localized state just above the Fermi energy. For each of these cases, the motion of the carrier is controlled by discrete steps between localized states. Scher and Montroll ${ }^{3}$ proposed that there should be a distribution in the hopping times (the time that the carrier spends between trapping events at localized states). From photo-current experiments, they proposed that the distribution of hopping times should follow an inverse power law:

$$
\psi(t)=\text { Const } \times t^{-\alpha}
$$

where $1 \geq \alpha \geq 0$ and $t$ is the hopping time. This functional behavior was also successfully derived assuming a continuous-time random walk for the motion of the carriers between trapping sites. 


\section{Electron Spin Resonance}

Electron Spin Resonance (ESR) spectroscopy 21,22 has a wide variety of applications in the study and classification of materials. It can be used to detect transition metals, free radicals, coupled radicals, lattice defects and more. Any system which contains or is composed of paramagnetic species in sufficient quantity can be detected by ESR.

\subsection{ESR Theory}

The resonance effect observed in ESR is duc to the interaction of a magnetic field with magnetic dipoles. Both electron angular momentum and nuclear angular momentum give rise to magnetic dipoles in matter. Approximately $99 \%$ of the electron angular momentum is due to the "spin" of the electron. Similarly, the nuclear spin accounts for the largest portion of the nuclear angular momentum. The Hamiltonian for the interaction of a magnetic field with matter can be written as:

$$
\begin{gathered}
H=\beta_{e} \vec{S} \cdot g_{e} \cdot \vec{H}_{m}+\beta_{n} \vec{I} \cdot g_{n} \cdot \vec{H}_{m}+\hbar \vec{S} \cdot A \cdot \vec{I}+\vec{S}_{1} \cdot B \cdot \vec{S}_{2} \\
=H_{e z}+H_{n z}+H_{h f}+H_{s}
\end{gathered}
$$


where:

$$
\begin{aligned}
& \vec{H}_{m}=\text { magnetic field strength } \\
& \beta_{e}=\text { electronic bohr magneton } \\
& \beta_{n}=\text { nuclear bohr magneton } \\
& \vec{S}=\text { spin operator (electronic) } \\
& \vec{I}=\text { spin operator (nuclear) } \\
& \mathrm{g}, \mathrm{A}, \mathrm{B}=\text { tensors characterizing interaction }(3 \times 3) \\
& \hbar=\text { Planck's constant/2 } \pi \\
& H_{e z}=\text { electronic Zeeman interaction } \\
& H_{n z}=\text { nuclear Zeeman interaction } \\
& H_{h f}=\text { hyperfine interaction } \\
& H_{s}=\text { spin-spin interaction }
\end{aligned}
$$

The primary intcraction for ESR is that of the electronic Zeeman effect. This effect can be modeled classically by a magnetic dipole interacting with a magnetic field (Fig. 3.1) The interaction encrgy of this system is:

$$
U=-\vec{\mu} \cdot \vec{H}_{m}
$$

The magnetic moment of an electron can be modeled by a charged particle moving in a circular orbit about an arbitrary axis (z) (Fig. 3.2). For such a system,

$$
\vec{\mu}=\frac{i A}{c} \hat{z}
$$

where $i$ is the current through the loop, $A$ is the area of the loop, and $c$ is the speed of light (Gaussian units). The current through the loop can be solved for in the following manner: 


$$
i \cong \frac{\Delta q}{\Delta t}=\frac{\Delta q}{\Delta x} \frac{\Delta x}{\Delta t}=-\frac{e v}{2 \pi r}
$$

$e$ and $v$ are the electron's charge and velocity, respectively, and $r$ is the radius of the orbit. The magnetic moment of the dipole is then:

$$
\vec{\mu}=\left(\frac{-e v}{2 \pi c r}\right) \pi r^{2} \hat{z}=\gamma m_{e} r v \hat{z} \quad \gamma=\frac{-e}{2 m_{e} c}
$$

The constant $\gamma$ is the gyromagnetic ratio, $m_{e}$ is the electron mass, and $m_{e} r v \hat{z}$ is the $z$ component of the angular momentum $\left(\vec{l}_{z}\right)$. Therefore,

$$
\vec{\mu}=\gamma \vec{l}_{2}
$$

This can be extended to a quantum mechanical model by converting the angular momentum into a quantum mechanical operator $\vec{l}_{z} \rightarrow m_{l} \hbar$ where $\left(m_{l}=0, \pm 1, \pm 2, \ldots\right)$. The quantum mechanical dipole moment of the electron becomes:

$$
\mu=\gamma \hbar m_{l}=-\beta m_{l} ; \quad \beta=\frac{e \hbar}{2 m_{e} c}
$$

The constant $\beta$ is the elctronic bohr magneton. The interaction energy for the magnetic field with the dipole is now a quantized value.

$$
U=-\vec{\mu} \cdot \vec{H}=\beta_{e} m_{l} H_{z}
$$

However, as was previously mentioned, the electron spin angular momentum is the dominant contribution to the magnetic moment of the electron. Therefore, the interaction energy is determined by the spin angular momentum: 


$$
U=g \beta_{e} m_{s} H_{z}=H_{e z}
$$

where $\mathrm{g}$ is a $3 \times 3$ tensor which takes $l_{z}$ into account and is characteristic of a material's spin system and $m_{s}$ is the spin quantum number which is confined to positive and negative half integer steps.

$$
m_{s}=0, \pm \frac{1}{2}, \pm 1, \pm \frac{3}{2}, \ldots
$$

The Zeeman splitting of the electron spin states is the driving effect of ESR. In absence of a magnetic field, the electron spin energy states are degenerate. However, in the presence of a magnetic field, the degeneracy is broken giving rise to separate energy states determined by the spin quantum number (Fig. 3.3). The energy difference between the $m_{s}= \pm 1 / 2$ states is:

$$
\begin{gathered}
\Delta E=H_{e z}\left(m_{s}=\frac{1}{2}\right)-H_{e z}\left(m_{s}=-\frac{1}{2}\right) \\
=g \beta_{e}\left(\frac{1}{2}+\frac{1}{2}\right) H_{z} \\
=g \beta_{e} H_{z}
\end{gathered}
$$

If a photon of energy $\Delta E$ is incident upon the electron, then it can be absorbed. This absorption would cause the elcctron to make a transition from one spin state to the other. This resonance effect is what is measured by ESR. Microwaves of frequence $v=g \beta_{c} H_{r} / h$ are directed into the material. As the magnetic field is swept through the resonance valuc $\left(H_{r}\right)$, microwaves are absorbed giving rise to the ESR signal. Therefore, ESR measures the net photon absorption as a func- 
tion of magnetic field strength. A typical microwave frequency used for ESR is the $X-B$ and $(\sim 9.5 \mathrm{GHz})$. The value of $\mathrm{g}$ is of importance when attempting to identify a given paramagnetic state. This value is determined from an ESR spectrum by finding the magnetic field value at maximum microwave absorption. The $g$ value for a free electron is 2.0023 . For $\mathrm{Si}$ defects in a-SiN:H the value is approximately $2.003^{16,26}$.

\subsection{Hyperfine Interaction}

The hyperfine interaction is also important in electron spin resonance. This interaction arises due to the presence of net nuclear spin as well as net electronic spin. The energy for such an interaction is:

$$
H_{h f}=\hbar \hat{S} \cdot A \cdot \hat{I}=\hbar A m_{S} m_{l}
$$

where $\hbar, A, \hat{I}, \hat{S}$, and $m_{S}$ are the previously defined values and $m_{l}$ is the nuclear spin quantum number. With this interaction, the electron spin levels are split into two new levels due to the introduction of nuclear spin. Conservation of angular momentum allows transitions only between states where $\Delta m_{I}=0$ These interactions are independent of the magnetic field strength and therefore remain constant (Fig. 3.4). The hyperfine split level now give rise to an absorption peak at two different magnetic field values instead of one.

The independence of the hyperfine interaction from the magnetic field strength allows one to distinguish between spectra containing multiple 
paramagnetic species and spectra containing a paramagnetic specie with a nuclear spin. Each case would give a spectrum with multiple absorption peaks. However, when the microwave frequency is changed the hyperfine peaks will remain split by the same magnetic field value while the splitting of multiple species g-values will change (due to the required change in the resonance fields to compensate for the frequency change - Fig. 3.5).

\subsection{The ESR Specrometer}

The typical ESR spectrometer is made up of a klystron (microwave source), a resonance cavity, a magnet, and a microwave absorption detector (Fig. 3.6). The microwaves from the klystron propagate down waveguides to the microwave cavity. The detector can be phasèd for measuring either dispersion or absorption.

The resonance cavity is manually tuned with the sample in place by an iris to couple the microwaves to the cavity (Fig. 3.7). The purpose of the tuning is to match the impedence of the wavcguide to that of the microwave cavity. The sample is placed in the cavity to maximize the sample interaction with the microwave's magnetic field while minimizing the interaction with the micowave's electric field. This allows for maximum sensitivity during detection and minimizes any dielectric losses that are induced by the microwave's electric field. The cavity is oriented to align the magnetic field from the microwaves perpendicular to the field produced from the magnet. This allows the microwaves to induce transitions between the different spin states. 
A measure of microwave cavity sensitivity is its quality factor (Q). The $Q$ of a cavity is defined by

$$
Q=\frac{2 \pi(\text { energy stored per cycle })}{\text { energy dissipated per cycle }}
$$

The cavity used for the experiments was a $T E_{102}$ cavity and a $T E_{103}$ optical cavity. The optical cavity has a port that allows for $100 \%$ illumination of the samples in situ (in the cavity). The loaded Q values for the cavities used during the experiments are approximately 3,000 . Figure 3.8 is a diagram of the apparatus used for the experiments discussed in the following chapter. 


\section{Light-Induced Electron Spin Resonance in Hydrogenated Amorphous Silicon Nitride}

In this chapter, the effects of ultra-violet (UV) light on the density of neutrally charged defects $\left(D^{\circ}\right)$ will be investigated. Since these defects are paramagnetic, electron spin resonance is an ideal method for monitoring the defect level in the material. The ability of UV light to induce neutral defects is measured by light induced electron spin resonance (LESR). The LESR signal observed is proportional to the density of light-induced, neutral $\left(D^{\circ}\right)$ defects present in the sample. The illumination time dependence of the LESR signal level is presented for different monochromatic light energies. We have examined the kinetics and photon energy dependence for the photo-bleaching (reducing by light) and photo-production of the LESR signal. In addition to the ESR data, high frequence capacitance-voltagc (CV) measurements were performed on the same material by Kanicki et al ${ }^{17,31,38}$. These measurements monitor the density of the positively charged, light-induced defects in a-SiN: $\mathrm{H}$ in MNS (metalnitride-semiconductor) capacitors (the behavior of which, under monochromatic illumination, will be compared with the ESR results). Light-induced phenomena are of great significance when attcmpting to ascertain the origin of the light induced defects. 


\subsection{Experimental Procedures}

The samples used for thes studics are hydrogenated, amorphous silicon nitride thin films produced by plasma-enhanced chemical vapor deposition (PECVD) using ammonia and silane gas in a $12: 1$ ratio at $400^{\circ} \mathrm{C}$. The resulting nitride has a N/Si ratio of 1.6 and contains $31 \%$ atomic hydrogen. The samples were deposited upon a fused quartz substrate in thicknesses of $760 \mathrm{~nm}$ for electron spin resonance. For high frequency capacitance verses voltage measurements (CV), the nitride was incorporated into MNS capacitor.

Electron spin resonance spectroscopy (ESR) was performed on Bruker Instruments ER-200 and ES-300 spectrometers operating at X-Band microwave frequencies. Quantification of spins (the neutral defect density) was determined using a National Bureau of Standards ruby standard with non-saturating microwave powers $(0.1 \mathrm{~mW})$ and a 5 Gauss signal modulation. The ruby standard is particularly well suited for quantifying the a-SiN:H LESR signal due to the similarity in the characteristics of their ESR spectra (ruby sample $\Delta H_{p p}=13$ Gauss). This allows for a higher degree of accuracy when determining spin densities from double integration. The ESR samples consisted of 4-6 strips ( $2 \mathrm{~mm} \times 4 \mathrm{~mm}$ ) cut from 1 inch diameter fused quartz disks with the nitride deposited uniformly on the surface.

Illumination of the samples was performed using an IR filtered, Oriel (\#66023) Xenon Deep U.V. lamp. The samples were held in an atmosphere of cool nitrogen for the room temperature studies. For the low temperature studies, the samples were contained in evacuated suprasil EPR sample tubes. 
Monochromatic illumination was obtained through the use of narrow bandpass filters (9-12 nm FWHM) and neutral density filters for intensity control. The light intensities were measured using OAI (model \#306) optical sensors set for 220 $\mathrm{nm}, 254 \mathrm{~nm}, 310 \mathrm{~nm}$, and $365 \mathrm{~nm}$. Ail of the filiters were checked for accuracy with a Hewlett Packard photo-spectrometer over the range of 200-800 nm.

For all measurements, a background spectra was taken for the sample tubes, the quartz substrate, and the un-illuminated sample. This spectra was systematically subtracted off of the light-induced measurements taken later. A study of the fused quartz $\left(\mathrm{a}-\mathrm{SiO}_{2}\right)$ substrate revealed that there was a light-induced ESR (LESR) signal produced. However, this signal is on the order of $1-4 \%$ of the magnitude observed for the a-SiN:H LESR and is disregarded. For the photoproduction studies, all room tempcrature illumination occurs outside of the ESR cavities. The uncertainty in measurements due to sample re-positioning is $\sim 3-4 \%$. For the low-temperature photo-bleaching studies, illumination occurred in situ using a Bruker ER-4104 OR optical cavity and the previously mentioned U.V. lamp. This negated the repositioning error and held samples at a constant, monitored temperature. Photo-production data is obtained by exposing previously un-illuminated samples to a series of light exposures for varying periods of time until saturation of the LESR is observed's. This is repeated for a number of different photon energies and light intensities. The procedure for photobleaching of the LESR is performed in a similar manner. First, the background spectra is obtained for an un-illuminated sample. Afterwards, the sample is illuminated to a saturated LESR signal level using broadband (un-filtered) light. It requires $15 \mathrm{~min}$. of illumination to reach this saturated level. A spectra is then 
taken to obtain the initial LESR level that will be photo-bleached. The sample is then illuminated in the cavity over a range of times with monochromatic, bleaching light and scanned to find the new LESR level. To determine if a significant amount of defects were lost during the transfer of the sample from the point of illumination to the ESR cavity (the photo-production studies were not performed in situ), spectra were taken for in situ illumination. There was no detectable difference between spectra taken when the lamp was on and when the lamp had been turned off for one minute. Hence, the number of defects lost while transferring a sample to the ESR cavity is negligible.

\subsection{Production of the LESR}

The light-induced electron spin resonance (LESR) signal in a-SiN:H has been attributed to the presence of paramagnetic silicon dangling bonds. Specifically, these dangling bonds are in the neutral $\left(D^{\circ}\right)$ state which contains one unpaired electron which gives rise to the paramagnetism. The presence of the two other charged states $\left(D^{+}, D^{-}\right)$cannot be directly detected by ESR due to the diamagnetic nature of these defects. In a-SiN $\mathrm{N}_{1.6}: \mathrm{H}$ the LESR signal obtained at X-band has a characteristic g-value of $\sim 2.0030$ and a linewidth of $\sim 13.5$ Gauss. The lower g-value (as compared to a-Si:H) has been attributed to the presence of nitrogen atoms backbonded to the Si dangling bonds ${ }^{34}$ and the linewidth arises from unresolved super-hyperfine interactions.

With prolonged illumination times, it is possible to saturate the LESR signal $1^{17,32}$ produced by either broadband or monochromatic light. It is important to note, however, that the LESR saturation level obtained with monochromatic 
light depends strongly upon the incident photon energy (Fig. 4.1). A minimum photon energy of $\sim 3.5 \mathrm{eV}$ is needed to produce a LESR signal in a-SiN $\mathrm{N}_{1.6}: \mathrm{H}$ at room temperature. The density of neutral dangling bonds at saturation $\left(\mathrm{N}_{s}\right)$ in creases dramatically as the photon energy is raised to the optical energy gap level $(\sim 5.15 \mathrm{eV}$ for this material).

The kinetics of the LESR signal production are of particular importance when attempting to ascribe a microscopic process to the defect formation. Previous studies of the thermal annealing of thermally generated defects in a-Si: $\mathrm{H}$ led to the proposal of a time dependent equilibration rate ${ }^{13}(v)$ in the time dependent rate equation of the thermally induced decay.

$$
\frac{d \Delta N}{d t}=-v \Delta N
$$

The equilibration rate follows a $\mathrm{t}^{-\alpha}$ which is generally associated with a dispersive diffusion ${ }^{3,15,18}$ process and has been previously observed in the drift mobility of excess charge carriers in amorphous photo-conductors. This time dependence has been attributed to a random walk or multiple trapping process in which a mobile species (such as atomic hydrogen or a mobile carrier) hops from site to site with a distribution of relcase times required for each succesive hop or trapping event. An equilibration rate constant of this form leads to a solution containing a stretched exponential ${ }^{18}$. The $\mathrm{t}^{-\infty}$ equilibration rate has recently been used by Redfield and Bube ${ }^{35}$ to describe the kinetics of the light-induced production of the 
LESR signal in a-Si:H. The rate equation for the production of neutral defects as described in Ref. 35 is:

$$
\frac{d N}{d t}=\left(\frac{t}{P}\right)^{-\alpha}\left[C_{1} R\left(N_{t}-N\right)-C_{2} R N\right]
$$

which can be simplified to

$$
\frac{d N}{d t}=\left(\frac{t}{P}\right)^{-\alpha}\{G-\gamma N\}
$$

where

$$
\begin{gathered}
G=N_{1} C_{1} R \\
\gamma=C_{2} R+C_{1} R
\end{gathered}
$$

$C_{1}$ and $C_{2}$ are the rate coefficients of lighi-induced carrier recombinations for the generation and bleaching of neutral defects, respectively. $R$ is the recombination rate for the photo-excited carriers which is proportional to the light intensity, and $N_{t}$ is the total number of states (or weak bonds) available for conversion into neutral dangling bonds. A recombination is any event in which a photo-excited carrier is trapped at a defect site (or at a weak Si-Si bond if bond breaking is assumed). Figure 4.2 is a schematic diagram of the possible recombination events that may occur under illumination (from ref. 22). In this diagram the transitions are:

1) $\mathrm{B}_{p}^{\circ:} D^{\circ}+$ hole $\rightarrow D^{4}$ 
2) $\mathrm{B}_{n}^{o:} D^{o}+e^{-} \rightarrow D^{-}$

3) $\mathrm{P}_{n}^{+}: D^{+}+e^{-} \rightarrow D^{\circ}$

4) $\mathrm{P}_{\bar{p}}^{-}: D^{-}+$hole $\rightarrow D^{\circ}$

5) T: weak bond $+e^{-}$or hole $\rightarrow D^{\circ}$

The ' $B$ ' transitions $(1,2)$ would remove neutral defects from the gap. The probability of a recombination resulting in one of these transitions is represented by the constant $\mathrm{C}_{2}$ The ' $\mathrm{P}$ ' transitions $(3,4)$ are those that would produce a neutral defect (as would the ' $\mathrm{T}$ ' transition (5) if bond breaking takes place). The probability of a recombination producing a neutral defect is represented by $C_{1}$. The $t^{-\alpha}$ dependence may arise (as previously mentioned) from the involvement of multiple trapping in these recombination processes. Therefore, a photo-excited carrier would be trapped and re-excited a number of times before being trapped at a metastable gap state. The steady state level to which the defect levels would then approach under illumination would be determined by an equilibrium between the $C_{1}$ and $C_{2}$ processes.

Unlike Redfield and Bube, thermal rates are ignored in this rate equation. Although the thermal anneal rate does follow a $t^{-\alpha}$ equilibration rate, the power dependence $(\alpha)$ differs between the light induced and the thermally induced processes at room temperature (this is shown in room temperature decay data to be covered later). Therefore the solution to Eq. (4.2b) is: 


$$
N=N_{f}-\left(N_{f}-N_{i}\right) \exp \left[-\left(\frac{t}{\tau}\right)^{\beta}\right]
$$

where $\mathrm{N}_{f}$ is the final total defect density which is equal to $\frac{G}{\gamma}, \mathrm{N}_{i}$ is the initial defect density, $\mathrm{G}$ is the generation rate, $\beta=1-\alpha$, and $\gamma$ is the net photobleaching rate. It is important to note that $\mathrm{N}_{f}$ is independent of light intensity since,

$$
\begin{gathered}
N_{f}=\frac{G}{\gamma}=\frac{N_{1} C_{1} R}{C_{2} R+C_{1} R} \\
=\frac{N_{t} C_{1}}{C_{1}+C_{2}}
\end{gathered}
$$

Eq. (4.4) can be further simplified if only the light-induced defects which make up the LESR are taken into account. If $\mathrm{N}^{\circ}$ is the number of light induced defects only, then

$$
N^{o}=N-N_{i}
$$

and

$$
N^{o}=N_{s}^{o}\left[1-\exp \left\{-\left(\frac{t}{\tau}\right)^{\beta}\right\}\right]
$$


where $\mathrm{N}_{s}^{o}$ is the number of light-induced spins at the LESR saturation level. It is important to note that while $\mathrm{N}_{j}^{o}$ depends strongly upon the photon energy of the illumination (as seen in Fig. 4.1), it is found to be independent of the light intensity over the range of $1 \mathrm{~mW} / \mathrm{cm}^{2}-10 \mathrm{~mW} / \mathrm{cm}^{2}$. This suggests that only a distinct number of states (weak bonds, charged states, etc.) are accessible to given energy of light for conversion to a neutral dangling bond and that the functional dependence of the bleaching and production rates on light intensity are the same (as is assumed for the model). Hence, the only property to be influenced by changes in intensity is the time required to reach saturation, not the saturation level. The above equation is used to fit data obtained by illuminating with 4.88 $\mathrm{eV}$ and $4.15 \mathrm{eV}$ light over a range of illumination times (Fig. 4.3). The saturation levels $\left(\mathrm{N}_{\mathrm{s}}\right.$ ) for the $4.88 \mathrm{eV}$ and $4.15 \mathrm{ev}$ illumination are 2.08E18 and 1.06E18, respectively. The values of $\beta$ and $\tau$ are obtained using a least squares fit to the data (values obtained are in Table 1). The values obtained for beta are quite close to those obtained by Redficld and Bube for the photo-production of the LESR in a-Si:H $(\beta=0.45 \pm 0.05)$ using broadband light, which indicates that the process involved for each of these materials may be similar.

\subsection{Photo-Bleaching of the LESR}

When performing the photo-production studies of the LESR, it was noticed that $4.88 \mathrm{eV}$ light produced a higher level of defects then the broadband light from which it was filtered (fig 4.1). Therefore, some wavelengths of the broadband light must be inhibiting or actively bleaching the LESR signal. To discover which photon energies were responsible for the bleaching, an LESR was 
first produced by broadband illumination. The samples were then re-illuminated with filtered, monochromatic light in an attempt to reduce the LESR signal. A reduction in signal was observed when re-illuminating with photon energies of 2.0 $-4.7 \mathrm{eV}$. The level of bleaching saturates with illumination time at a value which is dependent upon the bleaching photon energy (fig. 4.4). The maximum level of photo-bleaching observed was achieved using light with photon encrgies of 3.67 $\mathrm{eV}$. This energy is just above the threshold required to produce a LESR signal in a-SiN:H. The LESR signal was reduced by a maximum of $67 \%$ over $110 \mathrm{hrs}$ of illumination using this light. However, over the same period of time in the absence of light, the LESR signal lost $\sim 28 \%$ of the signal intensity due to thermal annealing losses at room temperature. So, only $39 \%$ of the LESR depletion can be directly attributed to photo-blcaching at room temperature. Approximately $33 \%$ of the broadband LESR signal is unaffected by the photo-bleaching light and thermal loss. Thereforc, some of the neutral defect states are inaccessible to the $3.67 \mathrm{eV}$ light or are being populated by the competing photo-production process.

When bleaching the LESR signal, it was noticed that the linewidth became narrower as defects were bleached away (Fig 4.5). When the final bleached LESR spectrum was subtracted from the initial spectrum, a broad ( $\sim 15$ Gauss linewidth) LESR signal remained. This signal represents the neutral defect states that had been removed by bleaching. The broader linewidth indicates that there may be chemical or configurational differences between the states that bleach and those that are unaffected by bleaching light. Preliminary studies of the monochromatic, sub-gap light-induced LESR signals show a narrower linewidth than the broadband produced signal. This may indicate that the above-gap 
photon energy light present in the broadband may induce some of the broad linewidth defects.

After photo-bleaching, the sample can be re-illuminated with broadband light to restore the LESR to its previous level. Cycling between the photo-bleached level and the initial LESR level can be performed a number of times (we have cycled six times) without a noticcable in the LESR signal levels. The ability to cycle between these two levels suggests a multiple energy level system (Fig. 2.3). To produce a LESR signal (neutral dangling bonds), $\sim 3.5 \mathrm{eV}$ photons are required to induce a transition from the ground state. For photo-bleaching, the minimum energy of $\sim 1.8 \mathrm{eV}$ is needed to perturb the metastable state back into the ground state which causes a loss in the LESR signal intensity. As the photon energies increase beyond $3.5 \mathrm{eV}$, a clear competition between the bleaching and producing processes arises which can be seen when the energy dependence of the photo-production and the photo-bleaching are placed on the same graph (fig. 4.6). In section $I$ of the graph, photo-bleaching clearly is the dominant process. As the photon energy is raised above the $3.5 \mathrm{eV}$ (section II), the photons are capable of both bleaching and producing neutral defects. At photon energies above $4.7 \mathrm{eV}$ (section III) photo-production dominates and no photo-bleaching is observed. This does not indicate that bleaching no longer occurs at high photon energies, or photo-production at low photon energies; it only indicates that photo-production overwhelms the bleaching process.

One possible mechanism for the observed photo-bleaching effect may involve the light-induced shuttling of electrons and holes between the defect states and the valence or conduction bands. This mechanism has been previously suggested 
by Adler ${ }^{19,20}$ to explain the light-induced production of neutral defects in a-Si:H. In this model, the ground state consists of the diamagnetic, charged states $\left(D^{+}, D^{-}\right)$and metastable excited states are the neutral dangling bonds $\left(D^{\circ}\right)$. Production of the LESR signal consists of either exciting electrons from the valence band into the $\mathrm{D}^{+}$state to convert it into a paramagnetic $\mathrm{D}^{\circ}$ state or exciting an electron out of the $\mathrm{D}^{-}$state to convert it into the $\mathrm{D}^{\circ}$ state. These recombinations were presented (Fig. 4.2) except that in this model there are no bond-breaking recombinations. This would occur as a multiple trapping process as was previously suggested. Photo-bleaching can be explained in a like manner with electrons being excited into or out of the $\mathrm{D}^{\circ}$ state to convert it into the $\mathrm{D}^{-}$or $\mathrm{D}^{+}$, respectively. However, $33 \%$ of the LESR is not bleachable. These unbleachable neutral defects may have been produced through bond-breaking which are, for some reason, impervious to bleaching. It is also possible that certain levels of defects cannot be bleached because, at the higher photon energies required to access them, the production of neutral defects already occurs to a greater extent than the bleaching.

Another possible mechanism for the production and bleaching of the LESR signal would involve the re-arrangement of local bond configurations brought about by interactions with incident photons. In this model, the subgap light would actually create new dangling bonds by breaking $\mathrm{Si}-\mathrm{Si}$ weak bonds. This could occur through the motion of hydrogen as suggested by thermal annealing studies ${ }^{15,18}$ of a-Si:H or by some other dispersive/diffusive process. The ground state would consist of centers which are in a configuration that strains the bond between the Si atom and its neighbor. Upon illumination with the proper wavelength of light, these sites can be converted into metastable dangling bonds 
(charged or neutral). Photo-bleaching will occur if these states are subsequently perturbed (with photons of the proper energy) out of the metastable state. These perturbed states would then relax back into the ground state with the broken bond reforming. This would result in the observed decrease of the LESR signal with photo-bleaching.

The previous rate equation (Eq. 4.2a) for the photo-production of the LESR signal can be expanded to describe the photo-bleaching phenomenon with a minor alteration. Allowances must be made for the neutral defect states that are not affected by the photo-bleaching light. The new rate equation is as follows:

$$
\frac{d N}{d t}=\left(\frac{t}{P}\right)^{-\alpha}\left[C_{1} R\left\{N_{t}-\left(N-\mu N_{i}\right)\right\}-C_{2} R\left(N-\mu N_{i}\right)\right]
$$

which simplifies to

$$
\frac{d N}{d t}=\left(\frac{t}{P}\right)^{-\alpha}\left\{G-\gamma\left(N-\mu N_{i}\right)\right\}
$$

In Eq. $4.8 \mathrm{a}, \mu$ is the fraction of the initial defect level which is unaffected by the bleaching light, $N_{i}$ is the initial defect density (achicved by broadband illumination to saturation), $\mathrm{G}$ is the generation rate, $\mathrm{N}$ is the number of neutral defects present at time $t, \gamma$ is the photo-bleaching rate constant, and $P$ and $\alpha$ are coinstants characterizing the dispersive diffusion. The solution of this equation is: 


$$
N(t)=\mu N_{i}+\frac{G}{\gamma}-\left\{\frac{G}{\gamma}-\left(N_{i}-\mu N_{i}\right)\right\} \exp \left[-\left(\frac{t}{\tau}\right)^{\beta}\right]
$$

Where $\mathrm{G} / \gamma=\mathrm{N}_{s}$ (the saturated ncutral defect density that the photo-bleaching light is capable of producing). The above equation can be further simplified to:

$$
N(t)=N_{s}+\mu N_{i}+\left\{(1-\mu) N_{i}-N_{s}\right\} \exp \left[-\left(\frac{t}{\tau}\right)^{\beta}\right]
$$

normalizing with respect to the initial spin density $N_{i}$,

$$
\begin{gathered}
\frac{N(t)}{N_{i}}=\frac{N_{s}}{N_{i}}+\mu+\left(1-\mu-\frac{N_{s}}{N_{i}}\right) \exp \left[-\left(\frac{t}{\tau}\right)^{\beta}\right] \\
=(1-\mathrm{A}) \exp \left[-\left(\frac{t}{\tau}\right)^{\beta}\right]+\mathrm{A}
\end{gathered}
$$

(Eq. 4.11b) is used to fit the photo-bleaching data obtained by using $3.67 \mathrm{eV}$ light and $3.27 \mathrm{eV}$ light (at $\sim 0.1 \mathrm{~mW} / \mathrm{cm}^{2}$ ) to bleach the broadband saturated LESR signal (Fig. 4.7). The parameters $\beta, \tau$, and $A$ are given in table 2 . for the room temperature bleaching performed in situ at room temperaturc. The thermal losses at room temperature are also fitted with eq. $4.11 \mathrm{~b}$ (Fig. 4.8) with the fitting parameters included in table 2. This allows a direct comparison of the exponential parameter and justifies leaving the thermal rates out of the initial rate 
equation (due to widcly different values of $\alpha$ ). The photo-bleaching data taken at room temperature only covers the range from $.1 \mathrm{~min}-1000 \mathrm{~min}$. The reason for not considering longer illumination times is that thermal annealing losses at room temperature become significant at times greater than $\sim 1000$ min and add a large degree of error to the bleaching data.

In order to minimize the thermal decay of the LESR signal, a second set of photo-bleaching experiments was performed. For these experiments the samples were held at $100 \mathrm{~K}$ during the photo-blcaching (Fig.4.9). As seen in Table 2, the extent of the photo-bleaching is (within experimental error) independent of temperature. Also, $\beta$ aproaches the value for a true exponential behavior (1.0) and $\tau$ decreases as the temperature is lowered. This indicates that the bleaching process becomes more efficient and less dispersive with lower temperatures. This is in good agreement with a multiple trapping model since, at lower temperatures, one would expect fewer hopping events to occur before a carrier is finally trapped into a metastable state. So, the distribution of trapping times (as indicated by $\alpha$ ) becomes narrower and $\alpha$ decreases. Also, at lower temperatures, bond reconfigurations would be inhibited as well as atomic diffusion. So, the involvement of bond-breakage or atomic diffusion in the bleaching process is not likely even though local reconfigurations may occur at lower temperatures. 


\subsection{Charged Defect Production}

The behavior of the charged defect states when exposed to light are also of interest when examining possible models for the defect production and bleaching. Previous studies have shown that the LESR signal can be reduced by the injection of charges into a-SiN:H using corona bias techniques ${ }^{26}$. Neutral defects capture these charges to become diamagnetic and charged. Using high frequency capacitance-voltage $e^{5,6,14,26,28}$ measurements on a thin film MNS capacitor which incorporates a-SiN:H, the presence of fixed charge in the film was shown to correspond with the density of positively charged defects present in the material ${ }^{39}$ $\left(\mathrm{N}^{+}\right)$. Using this technique, the kinetics of the positively charged defect production can be studied under illumination.

If one assumes that charge shuttling between the defect states and the valence or conduction bands is responsible for the light-induced effects observed, then the total defect density in the material would remain a constant $\left(\mathrm{N}^{0}+\mathrm{N}^{+}+\mathrm{N}^{-}=\right.$ constant). Therefore, the neutral defects lost during photo-bleaching would result in the increase of that many charged defects. In a similar manner, the production of the LESR signal can only come about by the conversion of charged defects to neutral defects. Since the capacitance-voltage techniques only measure the positively charged defects, the kinetics equation to describe that value should follow a stretched exponential equation similar to that observed for the neutral defects.

The saturation level $\left(\mathrm{N}_{j}^{+}\right)$of the positively charged defect density under monochromatic illumination increases with the incident photon energy in a manner similar to that of the neutral defect density (Fig. 4.10), but of two magnitudes 
lower in value. If the Adler model is assumed for the light induced processes, information on the possible correlation energy of the neutral defect can be gained. Since the positive $\left(D^{+}\right)$defects increase with illumination, they cannot be the source (or ground state) of the $D^{\circ}$ defects created by monochromatic light. In other words, recombinations of electrons with $D^{+}$states are of low probability. Therefore, the negative $\left(D^{-}\right)$state would have to be the source for conversion into neutral defect states. If this is the case, then the neutral defect would have a negative correlation energy since it energetically prefers existing in the $D^{-}$ground state. However, if the defects are produced by bond-breaking, then no such statement can be made since weak bonds would necessarily be the ground state.

The kinetics of the light-induced, positive defect production follow the same stretched exponential function as the neutral defect production ${ }^{31}$ :

$$
N^{+}(t)=N_{s}^{+}\left[1-\left(\frac{t}{\tau}\right)^{\beta}\right]
$$

where $N_{s}^{+}$is the positive defect density $\left(\right.$per $\left.\mathrm{cm}^{3}\right)$ and $t$ is the illumination time. This equation has been used to fit the production data in Fig. 4.12 (parameters in Table 1). Direct comparison between the $\beta$ and $\tau$ values for the positive charge production and those of the neutral defect production may not be directly comparable since the a-SiN:H for the $\mathrm{CV}$ measurements is incorporated into a MNS device. However, the fact that each follows a stretched exponential gives strong evidence for a like production process involved for each. 


\section{Summary}

Neutral defects can be produced by UV illumination in amorphous silicon nitrides. The density of these defects can be reduced by re-illuminating with light of photon energies ranging from $1.8-4.7 \mathrm{eV}$. Positively charged defects can also be induced in the material by illuminating with light of the same energies required for photo-production of the LESR $(h v>3.5 \mathrm{eV})$. The kinetics for photoproduction of the neutral defects, the photo-bleaching of these defects, and the photo-production of the positively charged defects can each be modeled from the same initial rate equation. The $\mathrm{t}^{-\alpha}$ dependence for the rate of each is highly suggestive of a multiple trapping model. Also, the neutral defect density can be cycled between the saturated and bleached levels which indicates that the most likely process involved for these defects is charge trapping and not bond brcaking. However, approximately $33 \%$ of the neutral defects cannot be bleached. These unbleachable defects may differ chemically (or configurationally) from the bleachable defects since there is a significant difference in the linewidth between the two. The unbleachable defects may arise from bond-breaking. Another possibility for not being able to bleach these defect states is ihat at the higher photon energies required to bleach them, a significant number of charged defects are already being converted to neutral defects. Therefore, the photo-production processes overwhelm the bleaching effect (as seen in Fig. 4.6). In this case bond-breaking would not be responsible for the inability to photo-bleach.

An important observation in support of the charge trapping model is the temperature dependence of the photo-bleaching. The extent of the bleaching is 
temperature independent from $100 \mathrm{~K}$ to $300 \mathrm{~K}$. Also, the bleaching occurs more quickly at lower temperatures and the decay becomes less dispersive. This puts into doubt the involvement of bond reconfigurations (or breaking) for the photobleaching process and gives further support to the multiple trapping model. It would be expected that lower temperatures would inhibit any processes involving atomic motion and, hence, the photo-bleaching would occur more slowly if thesc reconfigurations were involved. However, for charge trapping the mobile carriers would thermalize more rapidly at lower temperatures and fewer excitation steps would be invoived before being trapped at a metastable state. This explains why the bleaching effect would become less dispersive and occur more quickly at lower temperatures.

If the multiple trapping model docs accurately describe the photo-processes involved, then the correlation encrgy of the neutral defects is (most likely) predominantly negative. There probably exists a distribution of correlation energies, however, and therefore there would be some defects with a positive $U$ giving rise to the initial ESR signal observed before illumination. The argument for negative correlation energy arises due to the fact that the densities of both the positive and neutral defect densities is observed to increase under illumination. If multiple trapping is involved, these light-induced defects states must originally have existed in the negative, doubly occupied state. Also, bleaching can be explained as the neutral defect state re-capturing an electron to convert back to the negative ground state.

The negative correlation energy argument also may enable the identification of the physical arrangement of the Si defect in a-SiN:H. If the $\mathrm{U}$ is indeed neg- 
ative, the defects are most likely dangling bonds. Theoretical calculations have shown that the dangling bonds in a-Si:H exist predominantly in a negative $U$ state and the floating bond would have a positive $U$. This, as previously stated, hinges on the multiple charge trapping model being correct (as the evidence seems to indicate). 


\section{Acknowledgements}

I would like to thank Dr. M. S. Crowder for instructing me on the ESR technique, the use of his ESR labs and the many helpful discussions on this problem and Dr. J. Kanicki for discussions on his experimental technique and its ramifications. Further thanks are extended to Dr. D. Redfield for explaining his rate equation, Dr. K. Arya for advisement and review, and Dr. K. Parvin for review of this work. 


\section{References}

'M. H. Brodsky, Amorphous Semiconductors, Springer-Verlag, N.Y., N.Y., 1985

${ }^{2}$ R. Zallen, The Physics of Amorphous Solids, Wiley-Interscience, N.Y., N.Y., 1983

${ }^{3}$ H. Scher and E. W. Montroll, Phys. Rev. B 12, 2455 (1975)

${ }^{4}$ S. T. Pantelides, Phys. Rev. B 57, 2979 (1986)

sS. Y. Lin, G. Lucovsky, and W. B. Pollard, J. Non. Crys. Sol. 66, 291 (1984)

6J. Robertson, J. Appl. Phys. 54, 4490 (1983)

${ }^{7}$ R. A. Street and D. K. Bieglesen, J. Non. Crys. Sol. 35, 651 (1980)

${ }^{8}$ H. Okushi, T. Furui, R. Banerjce, and K. Tanaka, Appl. Phys. Lett. 54, 439 (1989)

9P. G. Le Comber and W. E. Spear, Philos. Mag. B 53, L1 (1986)

10Z. E. Smith and S. Wagner, Phys. Rev. B. 59, 688 (1987) 
"R. Car, J. Kelly, A. Oshiyama, and S. T. Pantelides, Phys. Rev. Lett. 54, 360 (1985)

${ }^{12}$ Y. Bar-Yam and J. D. Joannopoulos, Phys. Rev. Lett. 56, 2203 (1986)

${ }^{13}$ D. L. Staebler and C. R. Wronski, Appl. Phys. Lett. 31, 292 (1977)

${ }^{14}$ M. Stutzman, W. B. Jackson, and C. C. Tsai, Phys. Rev. B 32, 23 (1985)

${ }^{15}$ W. B. Jackson, Phys. Rev. B 38, 3595 (1988)

${ }^{16}$ D. T. Krick, P. M. Lenahan, and J. Kanicki, Phys. Rev. B 38, 8226 (1988)

${ }^{17} \mathrm{D}$. Jousse and J. Kanicki, Appl. Phys. Lett. 55,1112 (1989)

18J. Kakalios, R. A. Street, and W. B. Jackson, Phys. Rev. Lett. 59, 1037 (1987)

${ }^{19}$ D. Adler, M. E. Eberhart, K. H. Johnson, and S. A. Zygmunt, J. Non. Crys. Sol. 66, 273, (1984)

${ }^{20}$ D. Adler, J. Phys. (Paris) C4, 3 (1981)

${ }^{21}$ G. E. Pake and T. L. Estle, The Physical Principles of Electron Paramagnetic Resonance, W. A. Benjamin Inc., Reading, Mass. (1973) 
${ }^{22}$ C. P. Poole, Electron Spin Resonance 2nd Ed., Wiley-Interscience, NY, NY (1983)

${ }^{23}$ D. K. Biegelsen, W. Jackson, and R. L. Weisfield, J. Non. Crys. Sol. 66, 139 (1984)

${ }^{24}$ R. Hezel, Sol. Stat. Elec. 24, 863 (1981)

${ }^{25}$ C. J. Ultee, Phys. Chem. 64, 1873 (1960)

${ }^{26}$ D. Jousse and J. Kanicki, Appl. Phys. Lett. 52, 445 (1988)

${ }^{27}$ N. Lustig and J. Kanicki, J. Appl. Phys. 65, 3951 (1989)

28J. Robertson and M. J. Powell, J. Appl. Phys. 44, 415 (1984)

${ }^{29}$ K. L. Ngai and Y. Hsai, Appl. Phys. Lett. 41, 159 (1982)

${ }^{30}$ M. S. Crowder, E. D. Tober, and J. Kanicki, Appl. Phys. Lett. (submitted for publication 4/90)

3'J. Kanicki, M. Sakaran, A. Gelatos, M. S. Crowder, and E. D. Tober, Appi. Phys. Lett. (submitted for publication $3 / 90$ )

32J. Shinar, R. Shinar, S. Mitra, and J. Y. Kim, Phys. Rev. Lett. 62, 2001 (1989) 
${ }^{33}$ A. E. Delahoy and T. Tonon, AIP Conference Proceedings \#157, ed. B. L. Stafford and E. Sabisky, 263 (1987)

${ }^{34}$ N. Ishii, S. Oozora, M. Kumeda, and T. Shimizu, Phys. Stat. Sol. 114, K111 (1982)

${ }^{35}$ D. Redfield and R. H. Bube, Appl. Phys. Lett. 54, 1037 (1989)

${ }^{36}$ W. B. Jackson, C. C. Tsai, and R. Thompson, Phys. Rev. B, 64, 56 (1990)

${ }^{37}$ W. B. Jackson, J. M. Marshall, and M. D. Moyer, Phys. Rev. B 39, 1164 (1989)

38J. Kanicki and D. Jousse, IEE Elec. Dev. Lett. EDL-10, 277 (1989)

${ }^{39}$ J. Kanicki, M. Sankaran, and A. Gelatos, Appl. Phys. Lett. (submitted for publication 3/90)

${ }^{40}$ R. Schorner and R. Hezel, IEEE Trans. Electron. Dev. ED-28, 1466 (1981)

${ }^{41}$ N. Lustig and J. Kanicki, J. Appl. Phys. 65, 3951 (1989)

${ }^{42}$ M. Katayama, H. Morimoto, S. Yasuda, T. Tanaka, and M. Hijikigawa, SID '88 Digest, p. 310 (1988)

${ }^{43}$ T. Chikamura, S. Hotta, and S. Nagata, Mater. Res. Soc. Symp. Proc. 95, 421 (1987) 
${ }^{45}$ M. J. Powell, J. A. Chapman, A. G. Knapp, I. D. French, J. R. Hughes, A. D. Pearson, M. J. Edwards, R. A. Ford, M. C. Hemings, O. F. Hill, D. H. Nicholls, and N. K. Wright, Proccedings of the SID 29/3, 227 (1988) 


\section{Figure Captions}

Figure 2.1 Density of States vs Energy for a) crystalline semiconductors b) amorphous semiconductors. The mobility edge indicates the transition for the localized tail states to the extended band states. (from ref. 2)

Figure 2.2 Diagram of a) under-coordinated silicon dangling bond b) overcoordinated silicon floating bond.

Figure 2.3 Two Level Energy Well System vs Configuration Coordinate $\mathrm{q}$ for the ground state and metastable state of the silicon defect.

Figure 2.4 Diagram of the multiple trapping processes (a) and the variable hopping processes (b) for charged carriers in amorphous semiconductors.

Figure 3.1 Interaction of a magnctic dipole (or moment) with a magnetic field.

Figure 3.2 Circular electron orbital and subsequent magnetic moment.

Figure 3.3 Zeeman split electron spin states.

Figure 3.4 Zeeman split electron spin states with hyperfine interaction due to nuclear spin. 
Figure 3.5 Comparison of multiple paramagnetic specie spectra with hyperfine spectra for different microwave frequencies $(v)$.

Figure 3.6 Schematic diagram of ESR spectrometer.

Figure 3.7 Schematic diagram of $\mathrm{TE}_{102}$ ESR cavity.

Figure 3.8 Schematic diagram of experimental set-up.

Figure 4.1 Production of the ESR (neutral defect density $\mathrm{N}_{s}^{o}+\mathrm{N}_{o}$ ) vs. Photon energy. $N_{o}$ is the the spin density prior to any illumination.

Figure 4.2 Recombination reactions of photo-excited holes and electrons. $\mathrm{D}^{\circ}, \mathrm{D}^{-}$, and $\mathrm{D}^{+}$indicate the defect charge state prior to recombination.

Figure 4.3 Photo-production of the LESR vs. Illumination Time. (•) $4.88 \mathrm{eV}$, (ㅁ) $4.15 \mathrm{eV}$. Fitted with eq. 7 .

Figure 4.4 Photo-Bleaching of the LESR vs. Photon Energy. 1-A is the fraction of saturated broadband light-induced spins that are bleached.

Figure 4.5 LESR spectra of bleaching phenomenon. a) broadband saturated LESR signal b) saturated LESR signal after bleaching with $3.67 \mathrm{eV}$ light for 110 hours. c) LESR signal of bleached defects (a. - b.) 
Figure 4.6 Photo-bleaching ( $\bullet$ vs photon energy and Photo-production $(\Delta)$ vs. photon energy. The values are normalized to the saturated, broadband LESR spin density.

Figure 4.7 Photo-Bleaching of Broadband LESR vs Illumination Time. ( $\Delta$ ) 3.67 $\mathrm{eV}$ bleaching photon energy, $(\bullet) 3.27 \mathrm{eV}$ bleaching photon energy. Fitted with Eq. $4.11 \mathrm{~b}$.

Figure 4.8 Thermaily induced decay of the broadband LESR at room temperature fitted with Eq. $4.11 \mathrm{~b}$.

Figure 4.9 Photo-bleaching of the LESR signal with $3.67 \mathrm{eV}$ light. $(\Delta)$ at $300 \mathrm{~K}$, $(\bullet)$ at $100 \mathrm{~K}$. Fitted with Eq. $4.11 \mathrm{~b}$.

Figure 4.10 Production of the LESR and Net Fixed Charge vs. Photon energy.

Figure 4.11 Production of the LESR and Net Fixed Charge vs. Illumination Time. (•) $4.15 \mathrm{eV}$ induced LESR, (घ) $4.88 \mathrm{eV}$ induced LESR, (0) $4.13 \mathrm{eV}$ induced positive charge, and $(\square) 4.88 \mathrm{eV}$ induced positive charge. 


\section{Table 1}

Table of parameters $\beta$ and $\tau$ found using least squares fit of Eq. 3.7 to the data in Fig. 3.2 and Fig. 3.10.

\begin{tabular}{|c|c|c|c|}
\hline \multicolumn{5}{|c|}{ LESR data $\left(\mathrm{D}^{\circ}\right)$} \\
\hline $\mathrm{h} v$ & $\mathrm{~N}_{\varsigma}^{\circ}\left(\mathrm{cm}^{-3}\right)$ & $\beta$ & $\tau$ \\
\hline $4.15 \mathrm{eV}$ & $1.06 \times 10^{18}$ & $0.43 \pm 0.05$ & $21.6 \mathrm{~min}$ \\
\hline $4.88 \mathrm{eV}$ & $2.08 \times 10^{18}$ & $0.48 \pm 0.05$ & $19.5 \mathrm{~min}$ \\
\hline \multicolumn{4}{|c|}{$\mathrm{CV}$ data $\left(\mathrm{D}^{+}\right)$} \\
\hline $\mathrm{h} v$ & $\mathrm{~N}_{\mathrm{+}}^{+}\left(\mathrm{cm}^{-3}\right)$ & $\beta$ & $\tau$ \\
\hline $4.13 \mathrm{eV}$ & $2.20 \times 10^{16}$ & $0.74 \pm 0.05$ & $6.88 \mathrm{~min}$. \\
\hline $4.88 \mathrm{eV}$ & $8.50 \times 10^{15}$ & $0.77 \pm 0.05$ & $10.8 \mathrm{~min}$ \\
\hline
\end{tabular}




\section{Table 2}

Table of parameters for photo-bleaching data achieved using a least squares fit to Eq 3.11b. The data is plotted in Figs. 3.7, 3.8, and 3.9. note: (1-A) is the fraction of removable/bleachable defects.

\begin{tabular}{|c|c|c|c|c|}
\hline \multicolumn{5}{|c|}{ Data for Fig. 3.7 (photo-bleaching) } \\
\hline $\mathrm{h} v$ & Temp & $(1-\mathrm{A})$ & $\beta$ & $\tau$ \\
\hline $3.27 \mathrm{eV}$ & $300 \mathrm{~K}$ & 0.33 & $0.45 \pm 0.05$ & $267.6 \mathrm{~min}$ \\
\hline $3.67 \mathrm{eV}$ & $300 \mathrm{~K}$ & 0.40 & $0.52 \pm 0.05$ & $97.8 \mathrm{~min}$ \\
\hline \multicolumn{5}{|c|}{ Data for Fig. 3.8 (thermal decay of LESR) } \\
\hline $\mathrm{n} / \mathrm{a}$ & $300 \mathrm{~K}$ & 0.34 & $0.35 \pm 0.05$ & $1465 \mathrm{~min}$ \\
\hline \multicolumn{5}{|c|}{ Data for Fig. 3.9 (temperature dependence of bleaching) } \\
\hline $3.67 \mathrm{eV}$ & $300 \mathrm{~K}$ & 0.38 & $0.51 \pm 0.05$ & $123.1 \mathrm{~min}$ \\
\hline $3.67 \mathrm{eV}$ & $100 \mathrm{~K}$ & 0.39 & $0.80 \pm 0.05$ & $35.4 \mathrm{~min}$ \\
\hline
\end{tabular}


Figure 2.1

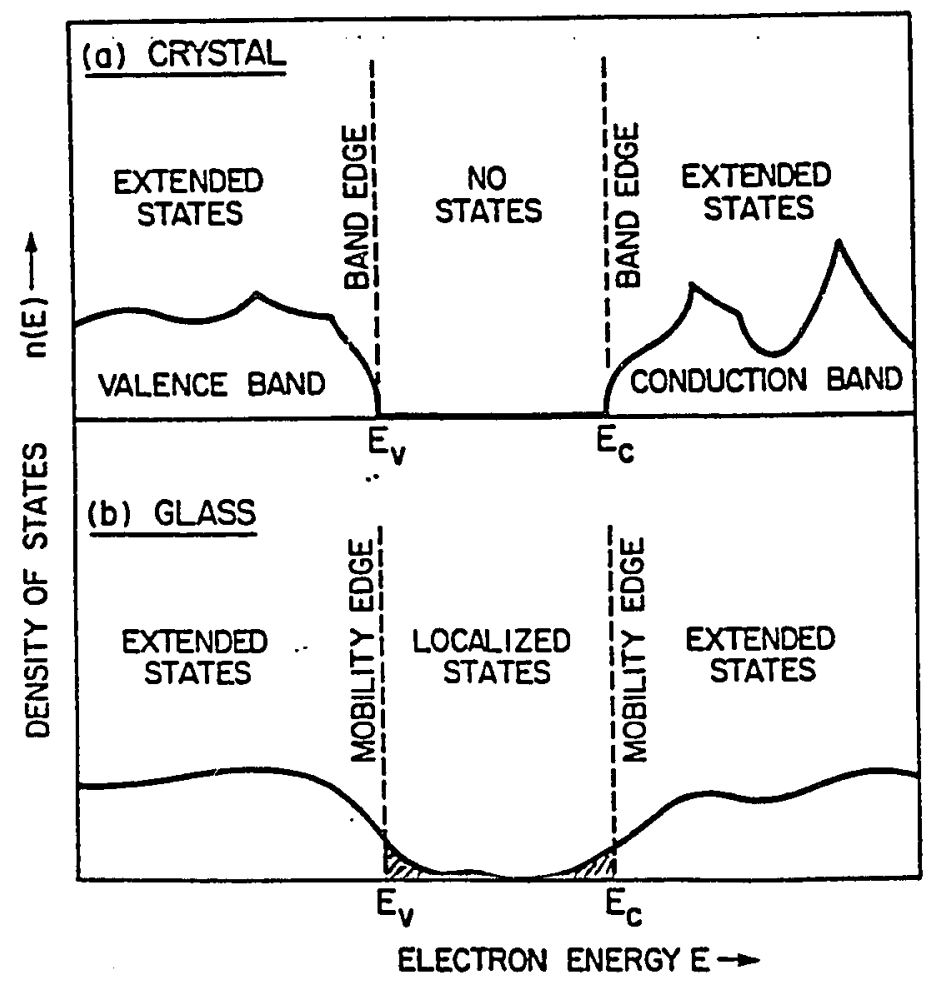


Figure $2 . \bar{\imath}$

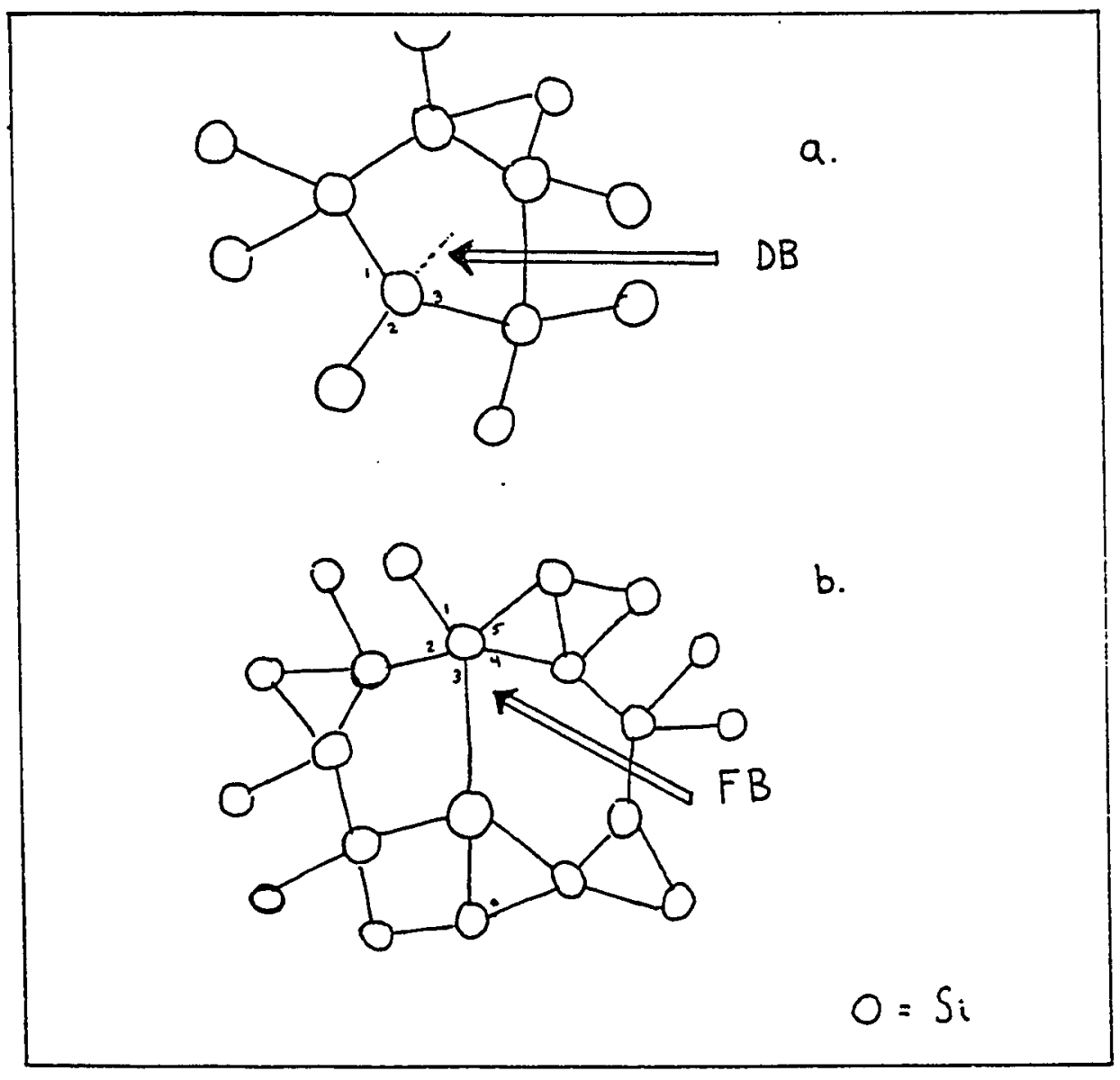


Figure 2.3

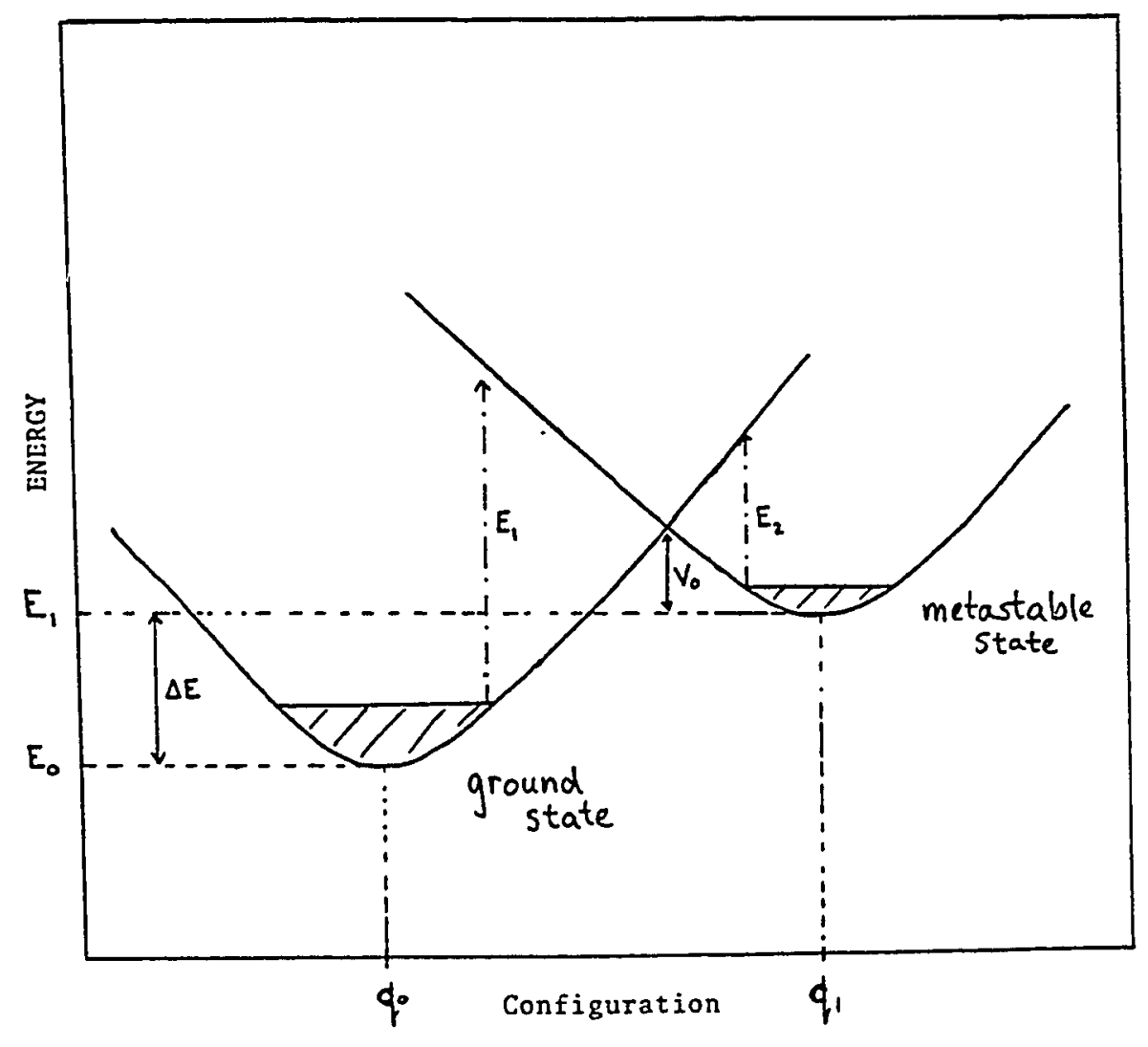


Figure 2.4

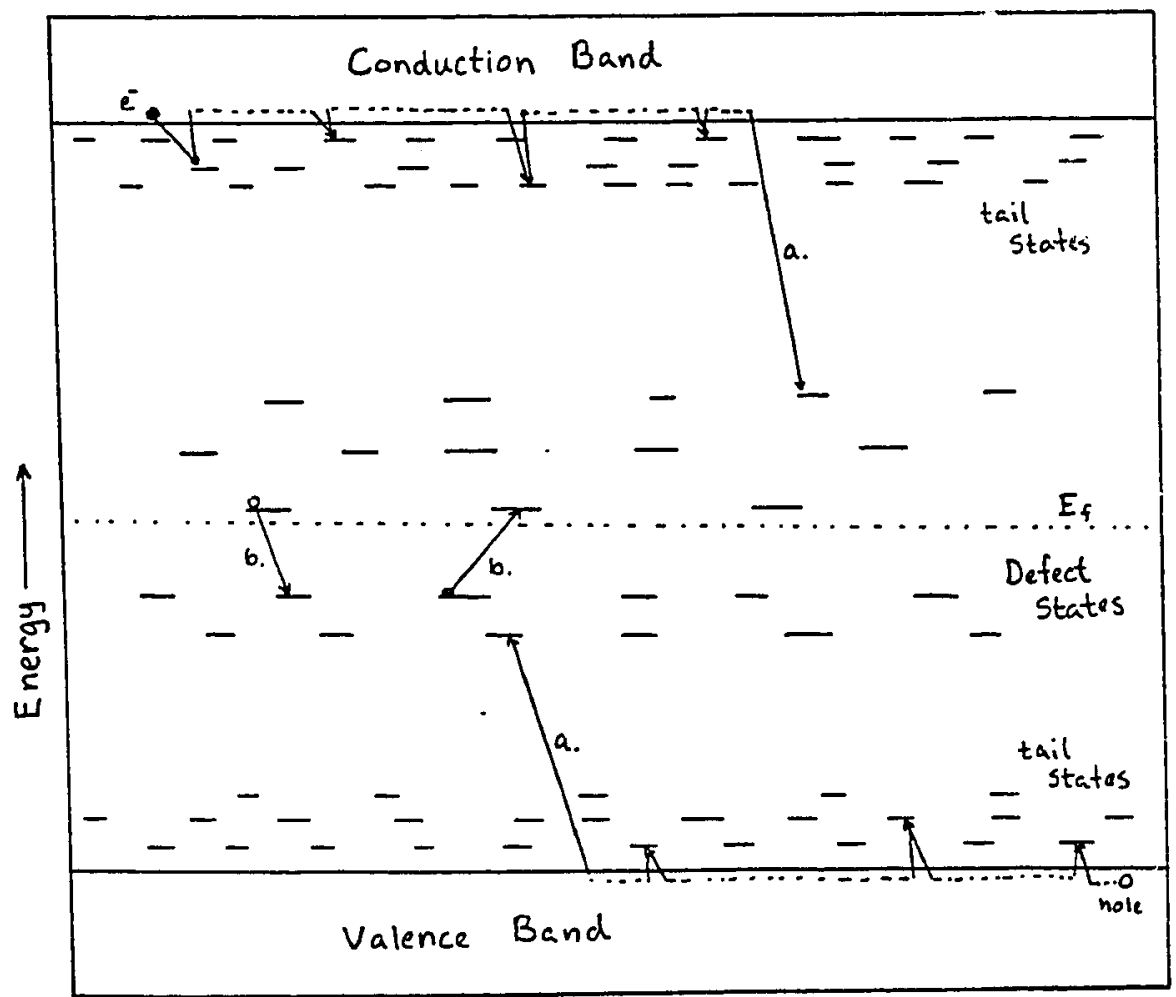


Figure 3.1

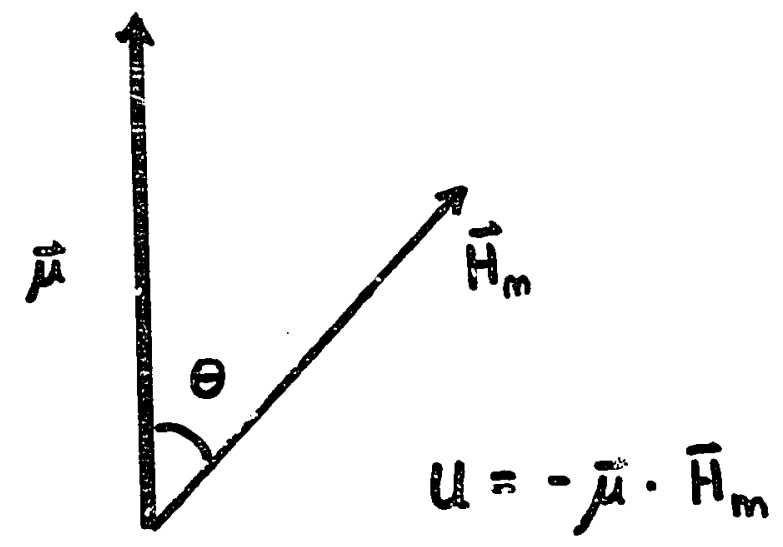


Figure 3.2

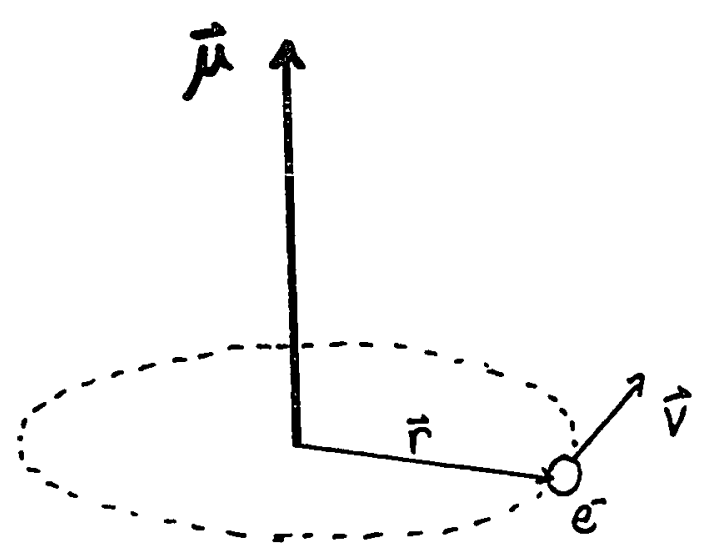


Figure 3.3

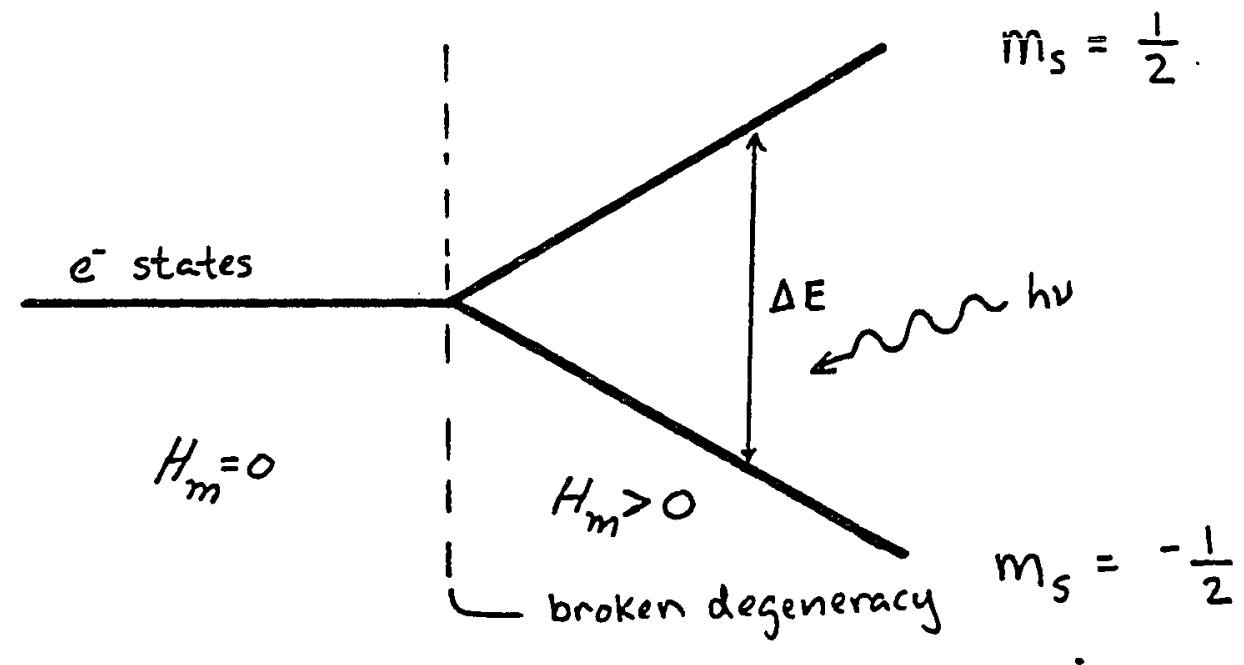


Figure 3.4

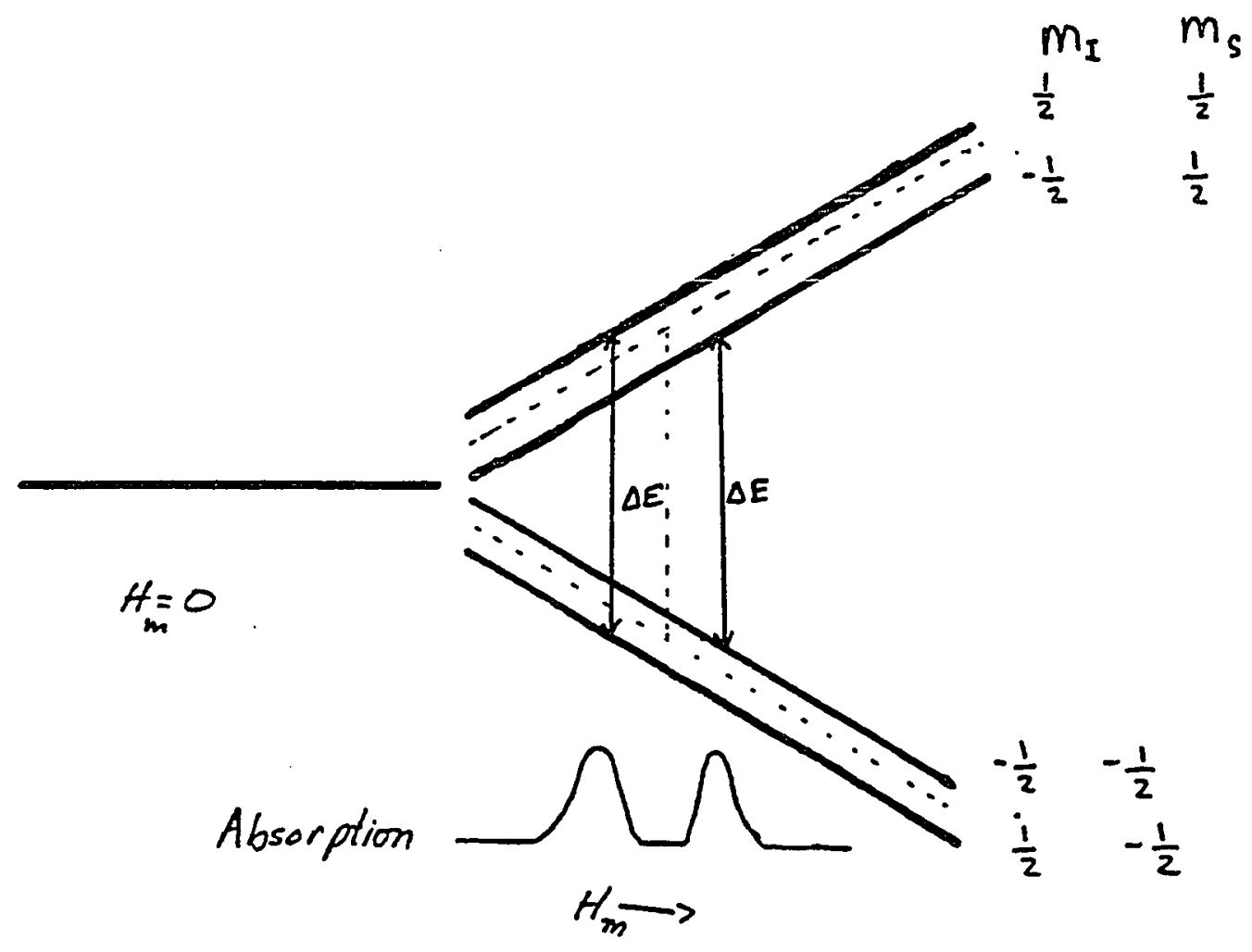


Figure 3.5

at $\nu=\nu_{0}$

Could be hyperfine or multiple specie.

$$
H_{m} \longrightarrow
$$

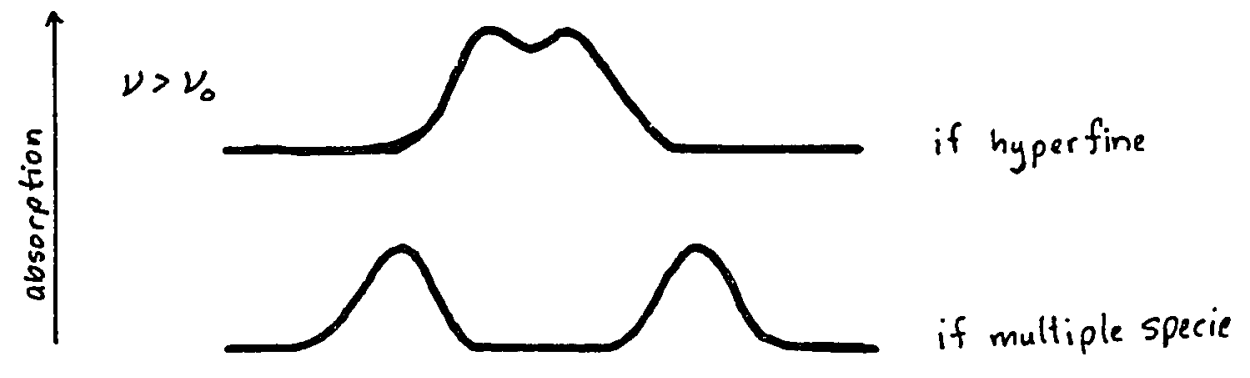

$$
\nu<\nu_{0}
$$

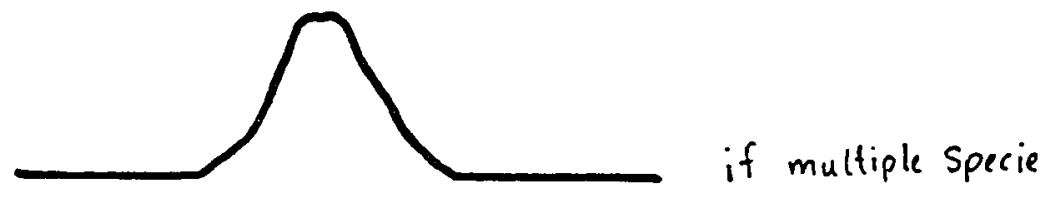


Figure 3.6

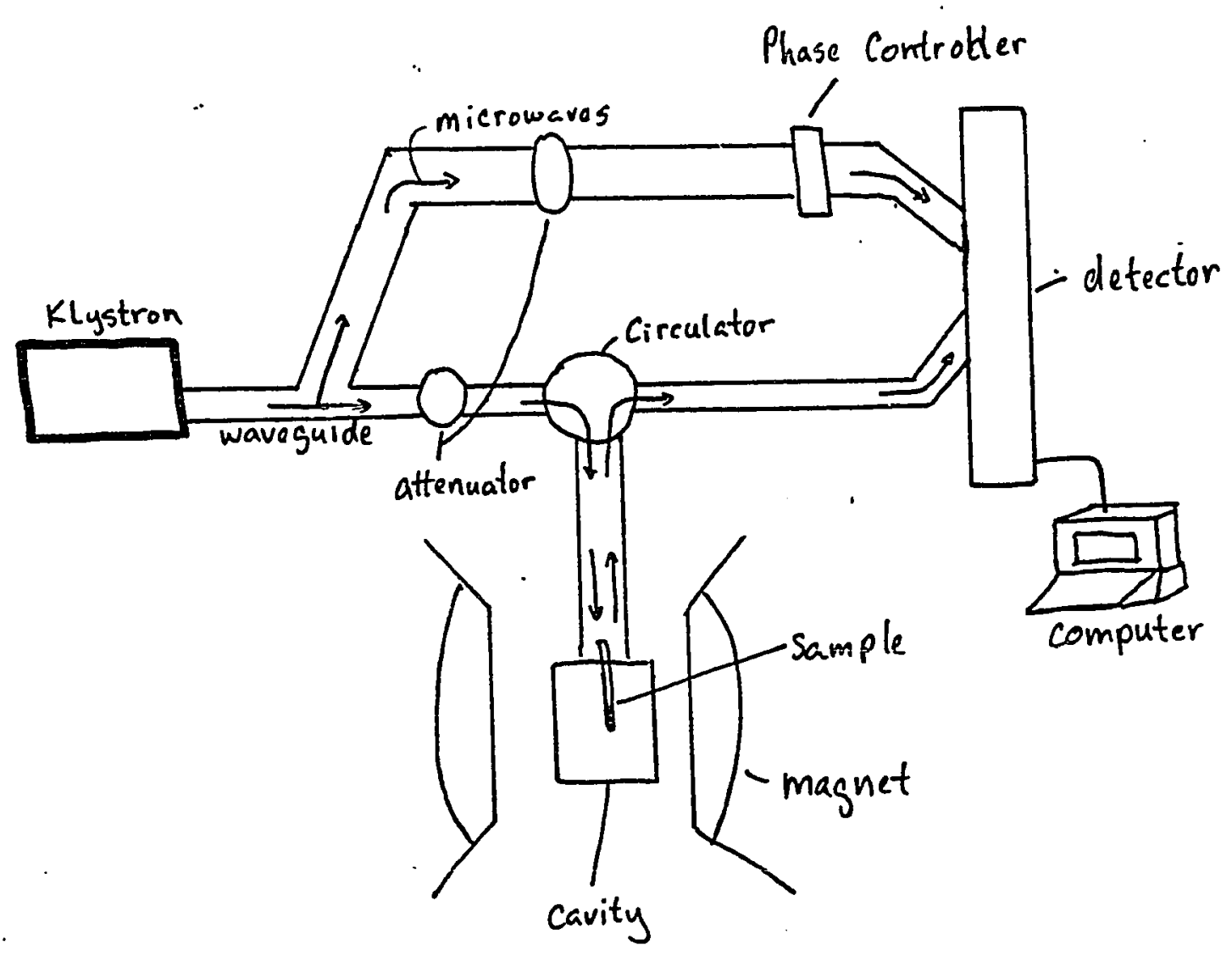




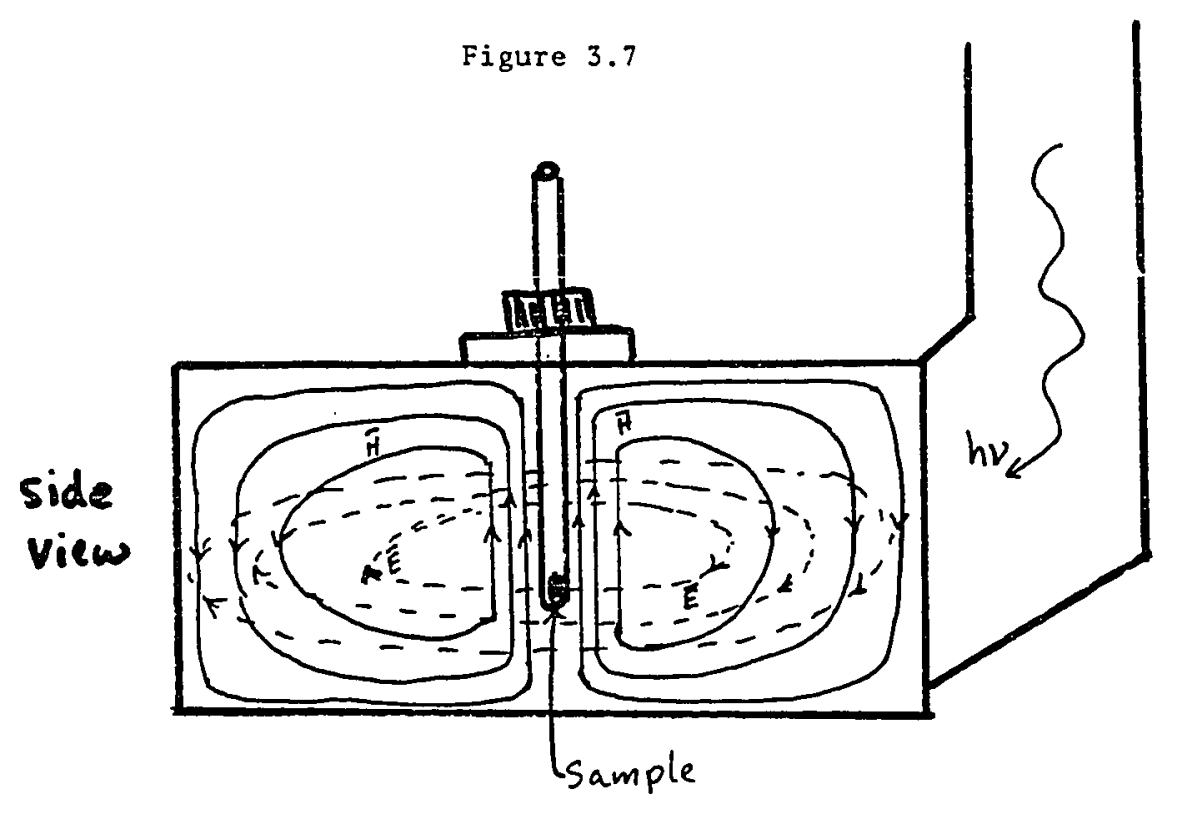


Figure 3.8

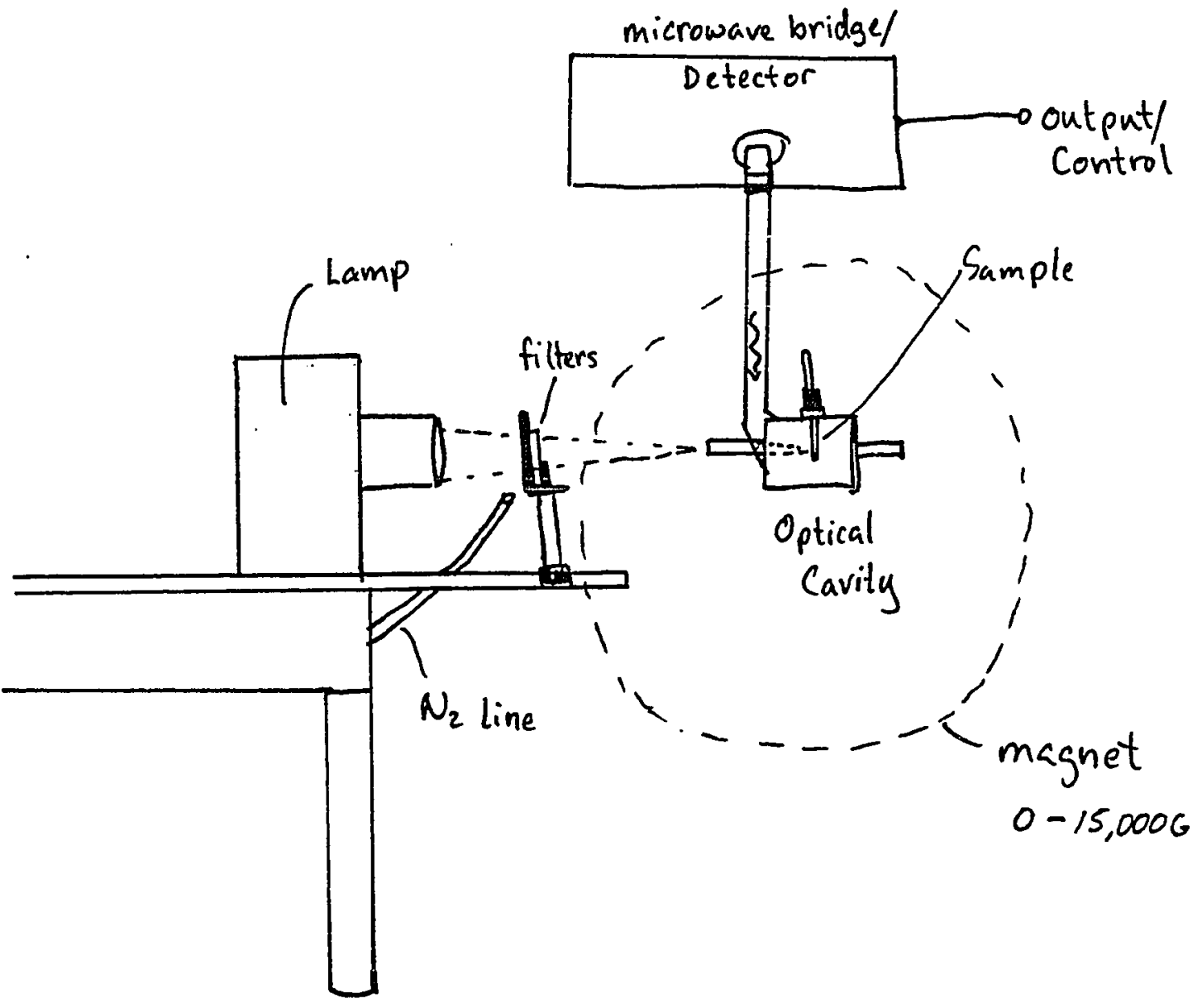


Figure 4.1

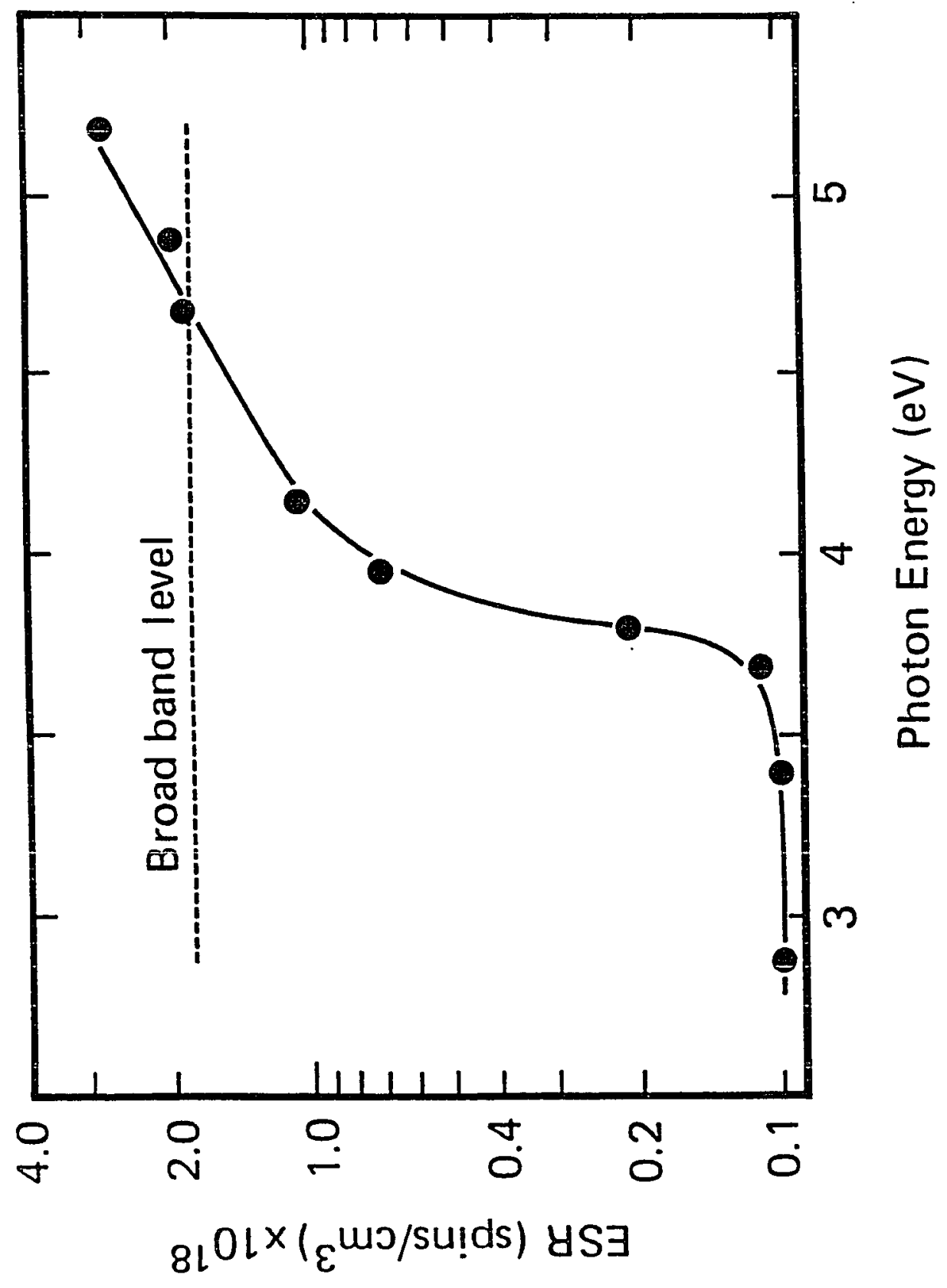


Figure 4.2

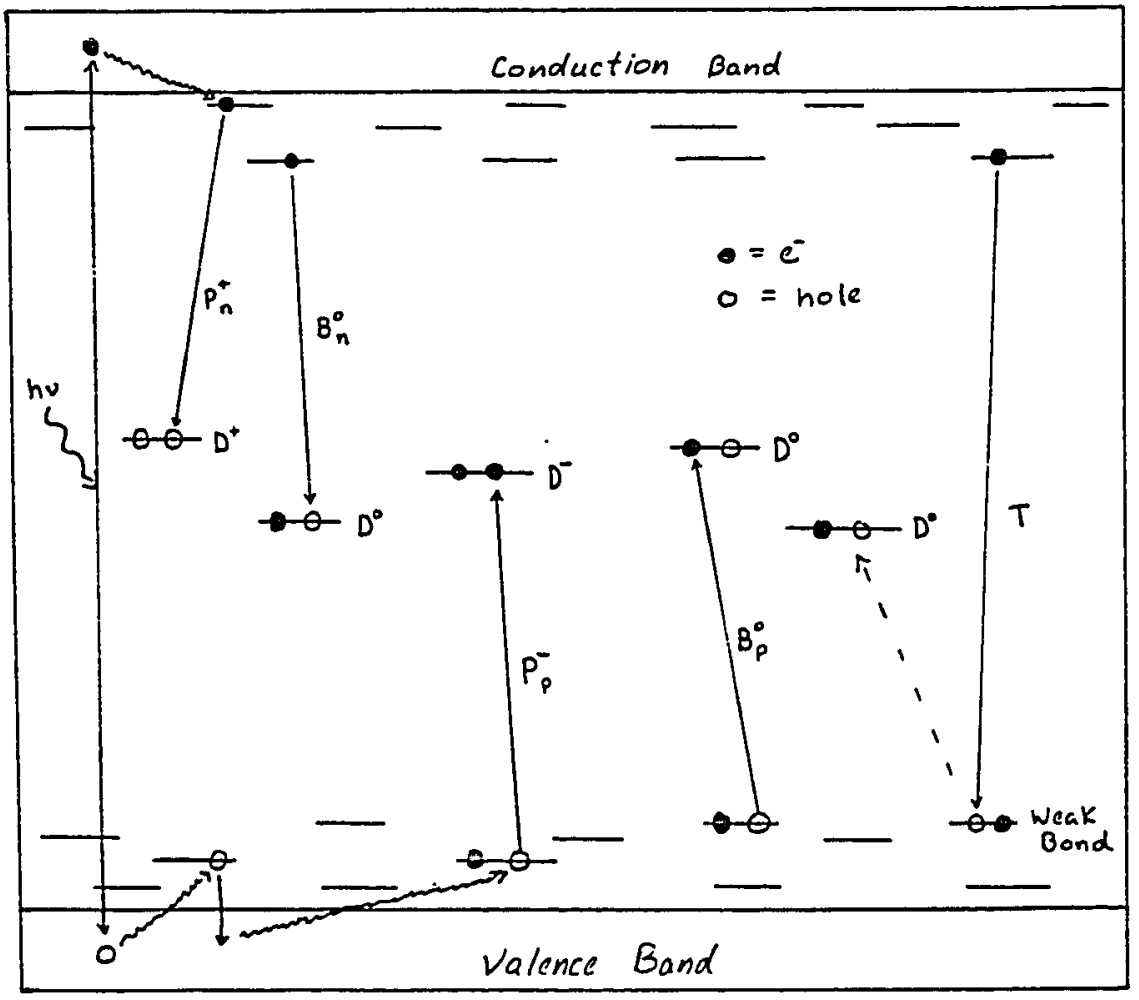


Figure 4.3

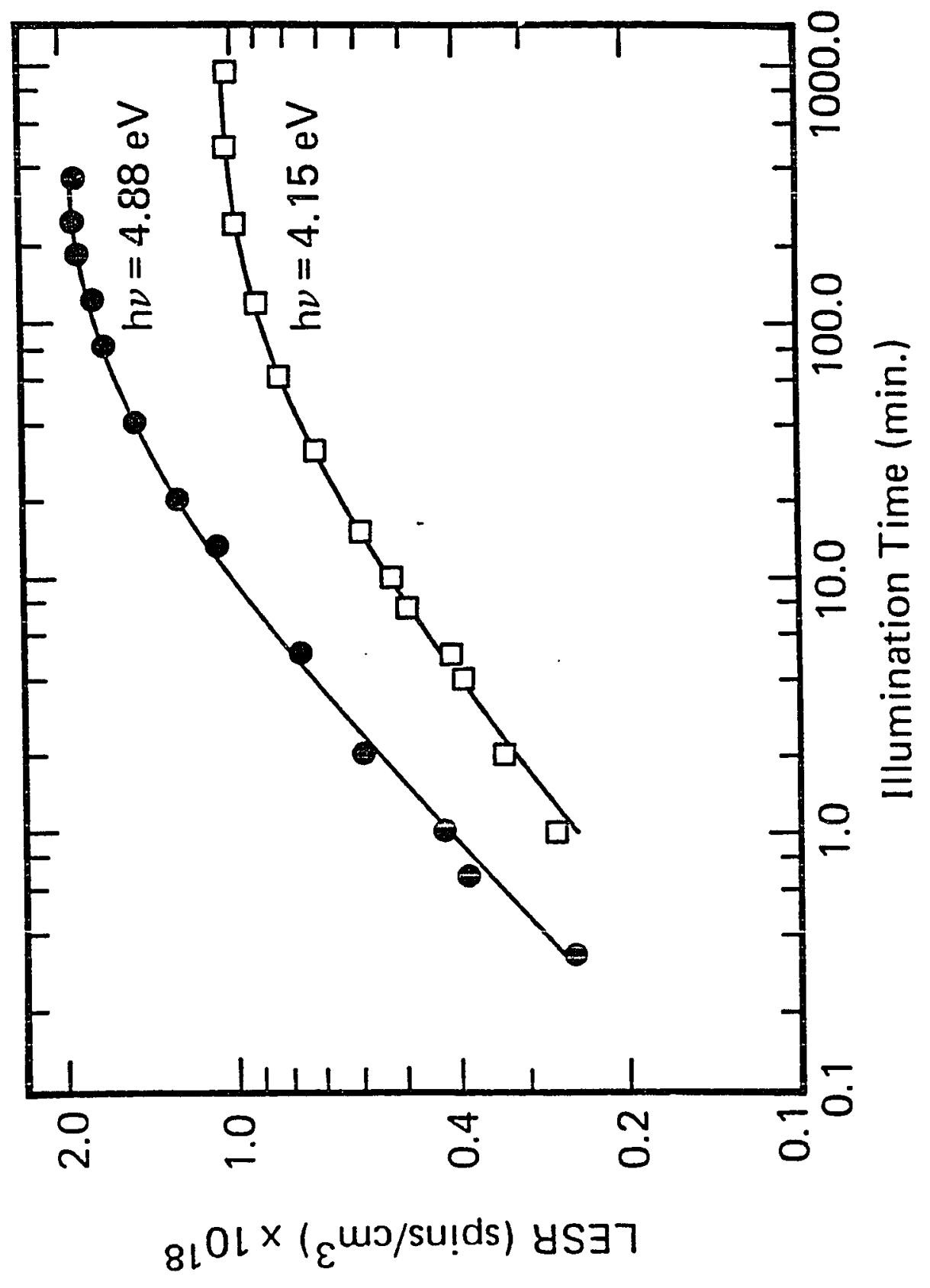


Figure 4.4

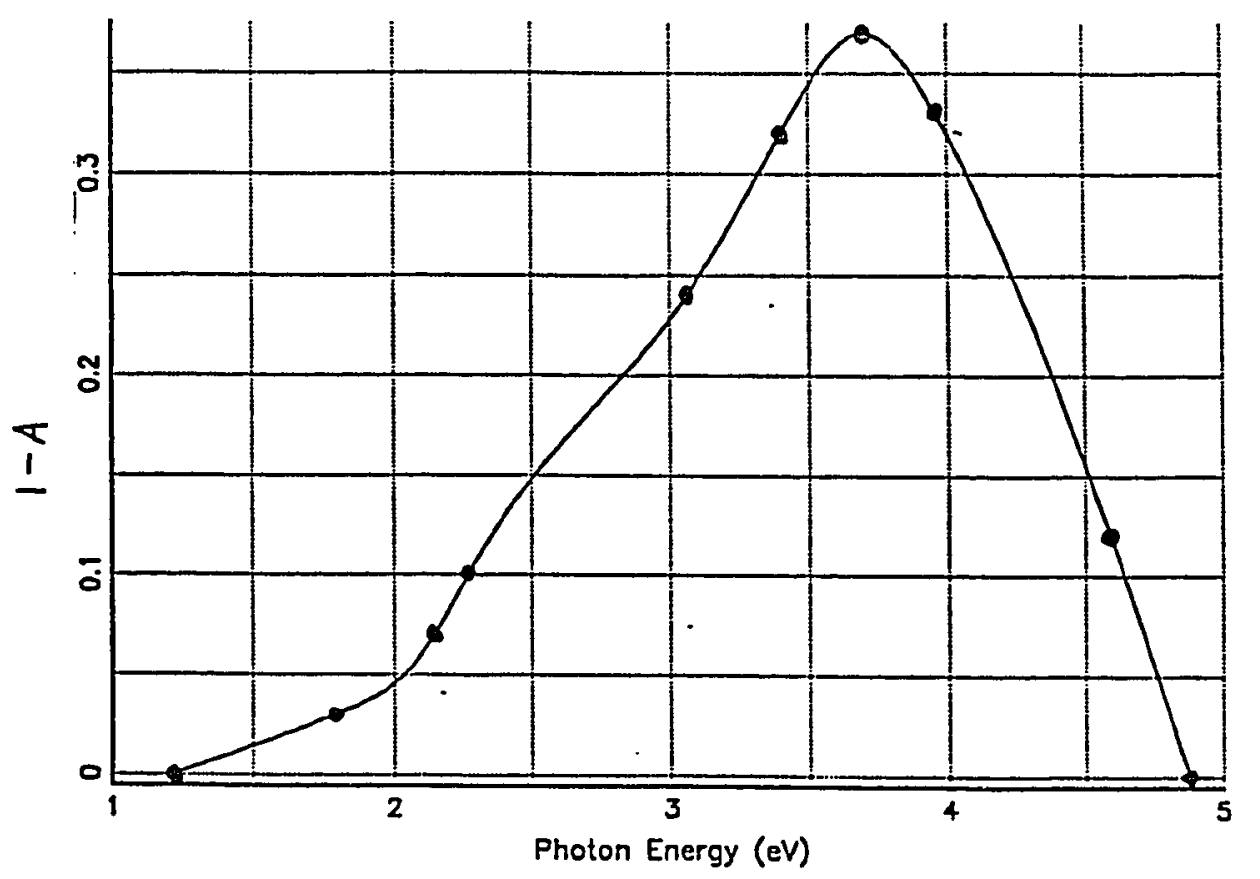

.. 
Figure 4.5

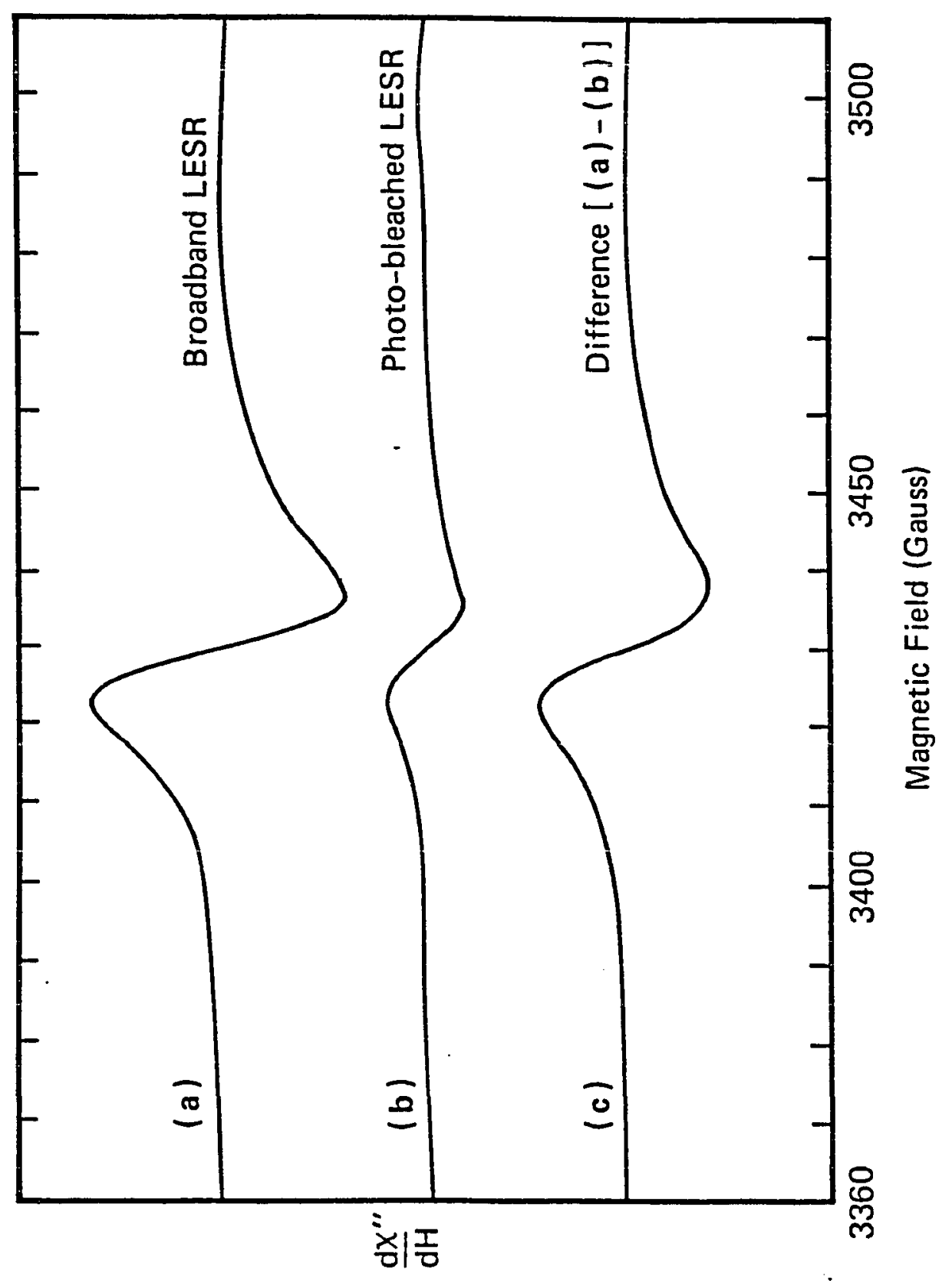




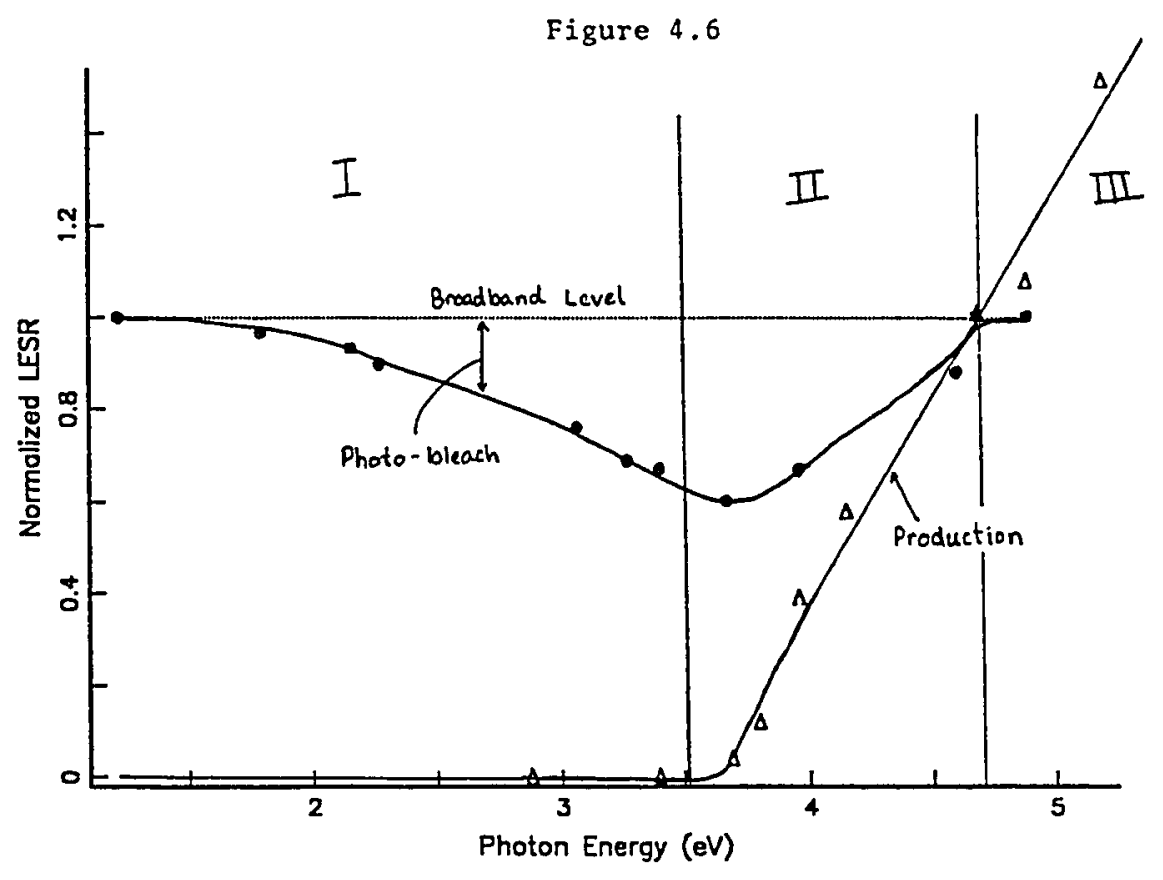




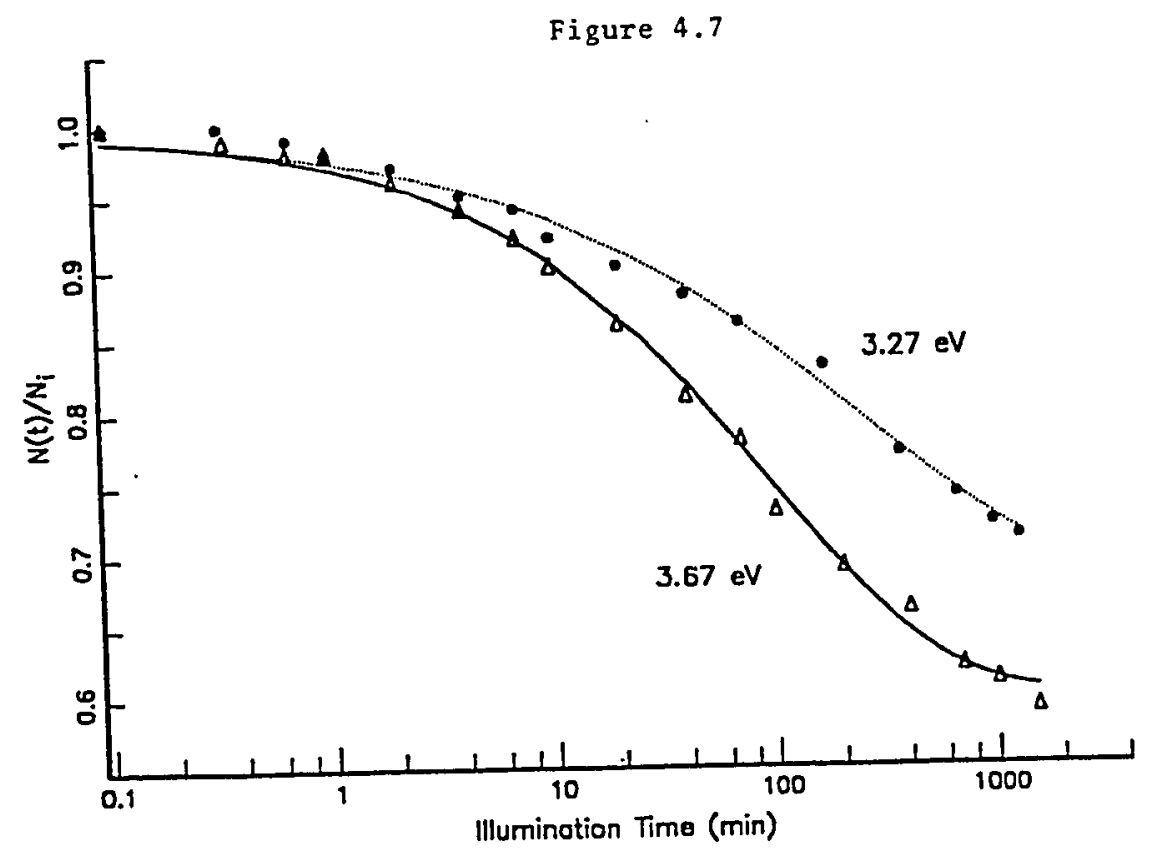




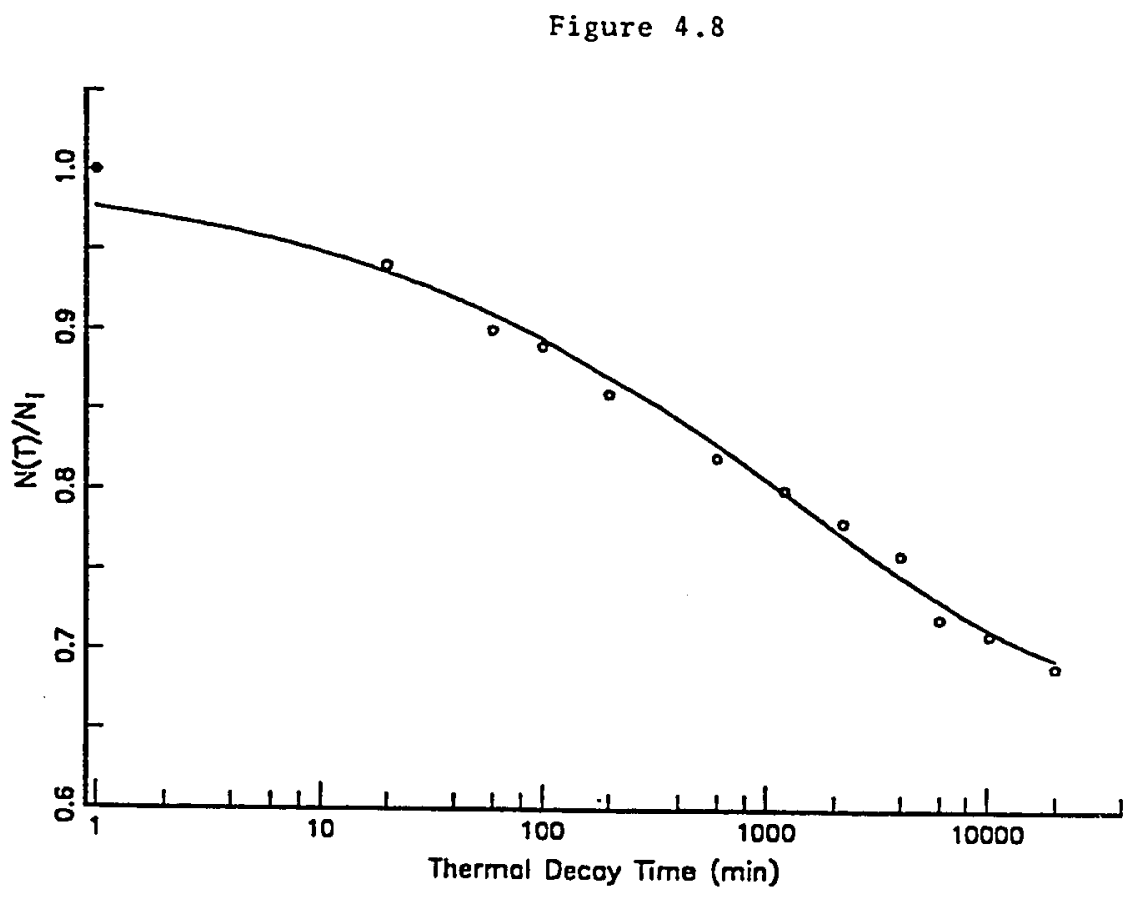


Figure 4.9

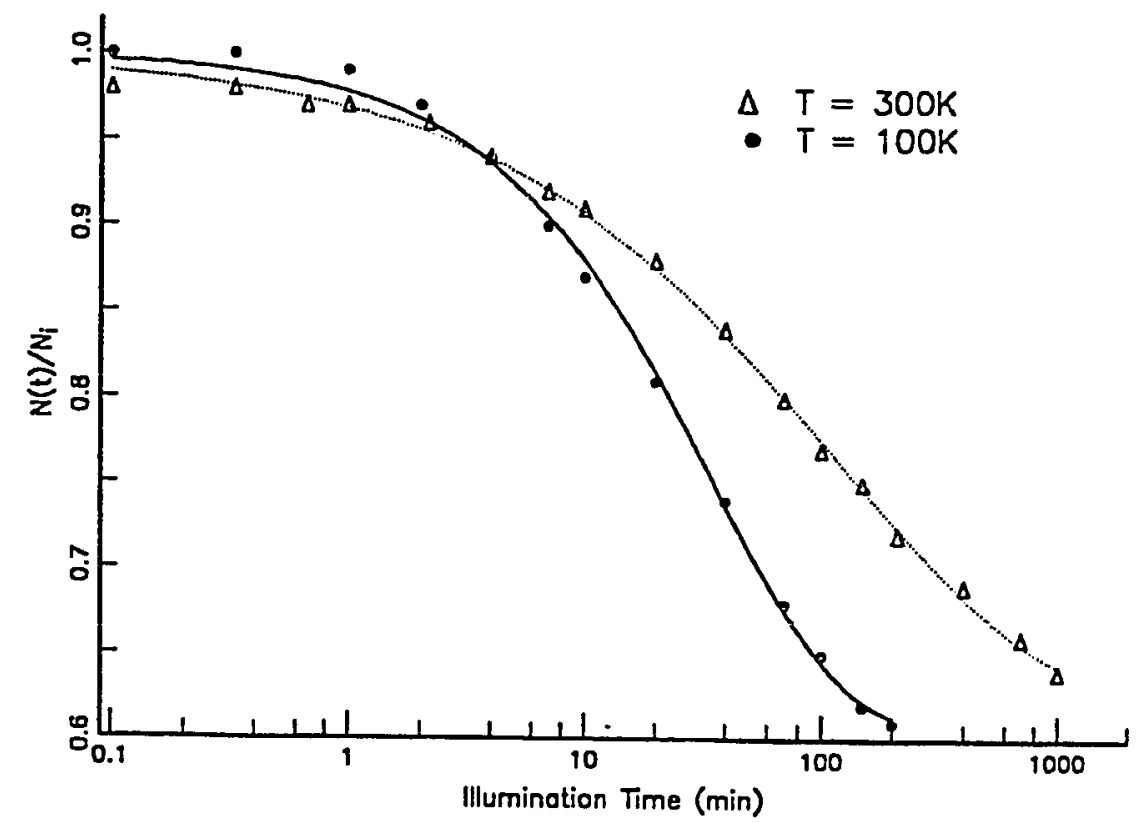


Figure 4.10

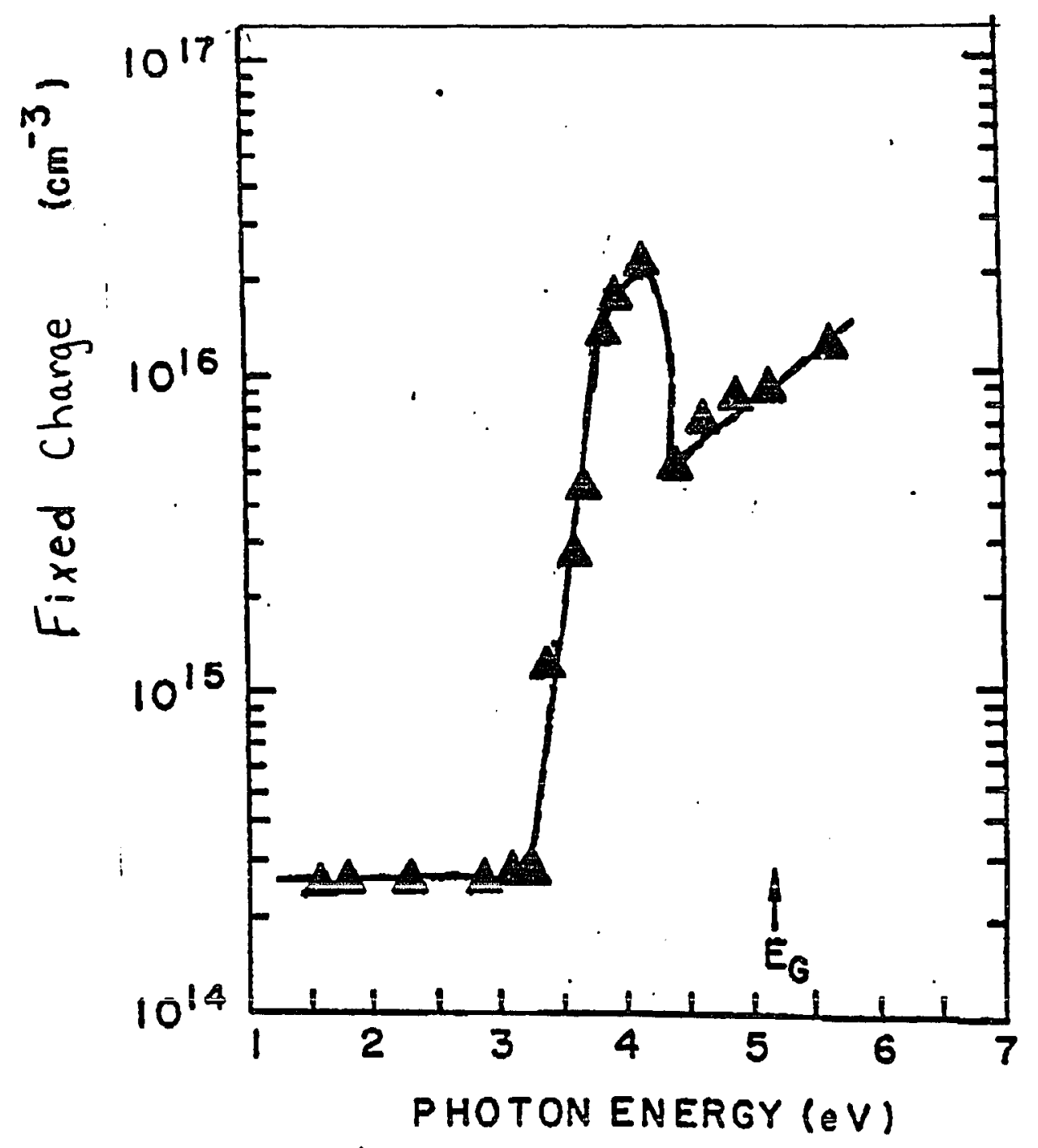


Figure 4.11

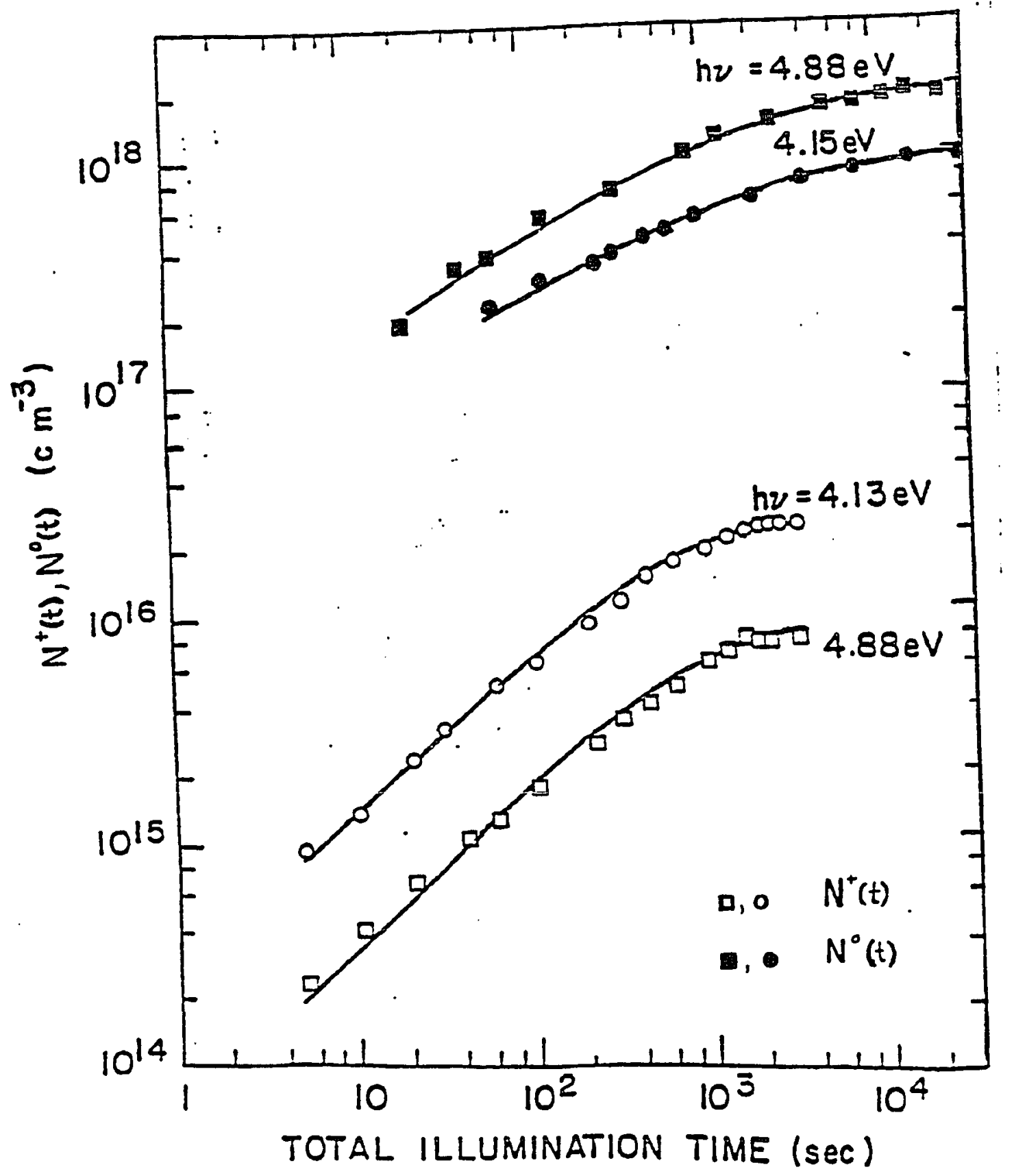

